TRANSACTIONS OF THE

AMERICAN MATHEMATICAL SOCIETY

Volume 362, Number 11, November 2010, Pages 5697-5727

S 0002-9947(2010)04973-6

Article electronically published on June 11, 2010

\title{
ELIMINATION OF RAMIFICATION I: THE GENERALIZED STABILITY THEOREM
}

\author{
FRANZ-VIKTOR KUHLMANN
}

\begin{abstract}
We prove a general version of the "Stability Theorem": if $K$ is a valued field such that the ramification theoretical defect is trivial for all of its finite extensions, and if $F \mid K$ is a finitely generated (transcendental) extension of valued fields for which equality holds in the Abhyankar inequality, then the defect is also trivial for all finite extensions of $F$. This theorem is applied to eliminate ramification in such valued function fields. It has applications to local uniformization and to the model theory of valued fields in positive characteristic.
\end{abstract}

\section{INTRODUCTION}

1.1. The main theorem. In this paper, we consider the defect (also called ramification deficiency) of finite extensions of valued fields. For a valued field $(K, v)$, we will denote its value group by $v K$ and its residue field by $\bar{K}$ or by $K v$. An extension of valued fields is written as $(L \mid K, v)$, meaning that $v$ is a valuation on $L$ and $K$ is equipped with the restriction of this valuation. Every finite extension $L$ of a valued field $(K, v)$ satisfies the fundamental inequality (cf. [En], $[\mathrm{Z}-\mathrm{S}]$ )

$$
n \geq \sum_{i=1}^{\mathrm{g}} \mathrm{e}_{i} \mathrm{f}_{i}
$$

where $n=[L: K]$ is the degree of the extension, $v_{1}, \ldots, v_{\mathrm{g}}$ are the distinct extensions of $v$ from $K$ to $L, \mathrm{e}_{i}=\left(v_{i} L: v K\right)$ are the respective ramification indices and $\mathrm{f}_{i}=\left[L v_{i}: K v\right]$ are the respective inertia degrees. Note that $\mathrm{g}=1$ if $(K, v)$ is henselian.

We say that $(K, v)$ is defectless in $L$ if equality holds in (1.1). Further, $(K, v)$ is called a defectless (or stable) field if it is defectless in every finite extension $L$ of $K$. Finally, $(K, v)$ is called a separably defectless field if it is defectless in every finite separable extension and called an inseparably defectless field if it is defectless in every finite purely inseparable extension of $K$. Note that every trivially valued field is a defectless field.

Every valued field of residue characteristic 0 is defectless; this is a consequence of the "Lemma of Ostrowski" (see Section 2.3). So we will always assume in the following that

$$
p=\operatorname{char}(\bar{K})>0 .
$$

Received by the editors May 27, 2008.

2010 Mathematics Subject Classification. Primary 12J10, 13A18; Secondary 12L12, 14B05.

(C)2010 American Mathematical Society 
Let $(L \mid K, v)$ be an extension of valued fields of finite transcendence degree. Then the following well-known form of the "Abhyankar inequality" holds (it is a consequence of Lemma 2.5 below):

$$
\operatorname{trdeg} L|K \geq \operatorname{rr} v L / v K+\operatorname{trdeg} \bar{L}| \bar{K},
$$

where $\operatorname{rr} v L / v K:=\operatorname{dim}_{\mathbb{Q}}(v L / v K) \otimes \mathbb{Q}$ is the rational rank of the abelian group $v L / v K$, i.e., the maximal number of rationally independent elements in $v L / v K$. We will say that $(L \mid K, v)$ is without transcendence defect if equality holds in (1.2).

In this paper, a function field will always mean an algebraic function field, where we include the case of finite algebraic extensions in order to avoid case distinctions. This paper is devoted to the proof of the following theorem, which has important applications.

Theorem 1.1 (Generalized Stability Theorem). Let $(F \mid K, v)$ be a valued function field without transcendence defect. If $(K, v)$ is a defectless field, then $(F, v)$ is a defectless field. The same holds with "inseparably defectless" in place of "defectless". If $v K$ is cofinal in $v F$, then it also holds with "separably defectless" in place of "defectless".

Take a valued field $(K, v)$ and fix an extension of the valuation $v$ to the separablealgebraic closure $K^{\text {sep }}$ of $K$. Then the absolute ramification field $K^{r}$, the absolute inertia field (also called strict henselization) $K^{i}$, and the henselization $K^{h}$ of $(K, v)$ (with respect to the chosen extension of $v$ ) are the ramification field, the inertia field and the decomposition field, respectively, of the extension $\left(K^{\mathrm{sep}} \mid K, v\right)$. Since all extensions of the valuation $v$ from $K$ to $K^{\text {sep }}$ are conjugate (i.e., are obtained from each other by composing with an automorphism of $K^{\mathrm{sep}} \mid K$ ), these fields are unique up to a valuation preserving isomorphism. Therefore, we will often speak of "the henselization" and work with $K^{h}$ without previously fixing an extension of the valuation. If $K^{h}=K$, then $(K, v)$ is called henselian. This holds if and only if the extension of $v$ from $K$ to every algebraic extension field is unique (implying that $\mathrm{g}=1$ in (1.1) ), or equivalently, if and only if $(K, v)$ satisfies Hensel's Lemma. An algebraic extension of a henselian field $(K, v)$ is called purely wild if it is linearly disjoint from $K^{r} \mid K$.

The algebraic closure of a field $K$ will be denoted by $\tilde{K}$. If $F \mid K$ is an arbitrary field extension and $L \mid K$ is an algebraic extension, then L.F will denote the field compositum of $L$ and $F$ inside of $\tilde{F}$.

Corollary 1.2. Let $(F \mid K, v)$ be a valued function field without transcendence defect, and $E \mid F$ a finite extension. Fix an extension of $v$ from $F$ to $\tilde{K} . F$. Then there is a finite extension $L_{0} \mid K$ such that for every algebraic extension $L$ of $K$ containing $L_{0},(L . F, v)$ is defectless in L.E. If $(K, v)$ is henselian, then $L_{0} \mid K$ can be chosen to be purely wild.

Theorem 1.1 was stated and proved in [K1]; the proof presented here is an improved version. The theorem is a generalization of a result of Grauert and Remmert $\mathrm{G}-\mathrm{R}$, which is restricted to the case of algebraically closed complete ground fields of rank 1 (i.e., with archimedean ordered value group). A generalization of the Grauert-Remmert Theorem was given by Gruson [G]. A good presentation of it can be found in the book $[\mathrm{B}-\mathrm{G}-\mathrm{R}]$ of Bosch, Güntzer and Remmert (§5.3.2, 
Theorem 1). The proof uses methods of nonarchimedean analysis. Further generalizations are due to Matignon and Ohm; see also $\mathrm{G}-\mathrm{M}-\mathrm{P}$. In [O], Ohm arrived independently of [K1] at a quite general version of the Stability Theorem. But like all of its forerunners, it is still restricted to the case $\operatorname{trdeg}(F \mid K)=\operatorname{trdeg}(\bar{F} \mid \bar{K})$ and is therefore not sufficient for the applications we will list below. Ohm deduces his theorem from B-G-R, Proposition 3, p. 215 (more precisely, from a generalized version of this proposition which is proved but not stated in $[\mathrm{B}-\mathrm{G}-\mathrm{R}]$ ).

In contrast to this approach, we give a new proof which replaces the analytic methods of $\mathrm{B}-\mathrm{G}-\mathrm{R}$, by valuation theoretical arguments. Such arguments seem to be more adequate for a theorem that is of (Krull) valuation theoretical nature. First, we reduce the proof to the study of Galois extensions of degree $p$ of special henselized function fields $F$. We deduce normal forms which allow us to determine that the extension is defectless. Related results can be found in the work of Hasse, Whaples, and Epp Ep, and in Matignon's proof of his version of Theorem 1.1 .

\subsection{Applications.}

- Elimination of ramification. This is the task of finding a (nice) transcendence basis $\mathcal{T}$ for a given valued function field $(F \mid K, v)$ such that for some extension of $v$ to the algebraic closure of $F$, the extension $\left(F^{h} \mid K(\mathcal{T})^{h}, v\right)$ of respective henselizations is unramified, that is, the residue fields form a separable extension $\bar{F} \mid \overline{K(\mathcal{T})}$ of degree equal to $\left[F^{h}: K(\mathcal{T})^{h}\right]$, and $v F=v K(\mathcal{T})$. (Note that passing to the henselization does not change the value group or residue field.) In K-K1] we use Theorem 1.1 to prove:

Theorem 1.3. Take a defectless field $(K, v)$ and a valued function field $(F \mid K, v)$ without transcendence defect. Assume that $\bar{F} \mid \bar{K}$ is a separable extension and $v F / v K$ is torsion free. Then $(F \mid K, v)$ admits elimination of ramification in the following sense: there is a standard valuation transcendence basis $\mathcal{T}=\left\{x_{1}, \ldots, x_{r}, y_{1}, \ldots, y_{s}\right\}$ of $(F \mid K, v)$ such that

a) $v F=v K \oplus \mathbb{Z} v x_{1} \oplus \ldots \oplus \mathbb{Z} v x_{r}$,

b) $\bar{y}_{1}, \ldots, \bar{y}_{s}$ form a separating transcendence basis of $\bar{F} \mid \bar{K}$.

For each such transcendence basis $\mathcal{T}$ and every extension of $v$ to the algebraic closure of $F,\left(F^{h} \mid K(\mathcal{T})^{h}, v\right)$ is unramified.

A standard algebraically valuation independent set in an extension $(F \mid K, v)$ of valued fields is a set $\mathcal{T}=\left\{x_{i}, y_{j} \mid i \in I, j \in J\right\}$ in $F$ where the values $v x_{i}, i \in I$, are rationally independent values in $v F$ modulo $v K$, and the residues $\bar{y}_{j}, j \in J$, are algebraically independent over $\bar{K}$. If $J$ is a basis of $F \mid K$, then it is called a standard valuation transcendence basis. In this paper, we deduce:

Corollary 1.4. Let $(F \mid K, v)$ be a valued function field without transcendence defect. Fix an extension of $v$ to $\tilde{F}$. Then there is a finite extension $L_{0} \mid K$ and a standard valuation transcendence basis $\mathcal{T}$ of $\left(L_{0} . F \mid L_{0}, v\right)$ such that for every algebraic extension $L$ of $K$ containing $L_{0}$, the extension $\left((L . F)^{h} \mid L(\mathcal{T})^{h}, v\right)$ is unramified.

- Local uniformization in positive and in mixed characteristic. Theorem 1.3 is a crucial ingredient for our proof that Abhyankar places of function fields (i.e., places for which the corresponding valued function field is without transcendence defect) admit local uniformization (cf. [K-K1, [K2], $\mathrm{K} 3$ ]), provided 
that a (necessary) separability condition for their residue field extension is satisfied. Together with a theorem proven in [K8], it is also the main ingredient for local uniformization of arbitrary places after a finite extension of the function field $([\mathrm{K}-\mathrm{K} 2])$. The analogous arithmetic cases (also treated in [K-K1] and [K-K2]) use Theorem 1.1 in mixed characteristic. The proofs solely use valuation theory.

Let us mention at this point that our approach has some similarity with S. Abhyankar's method of using ramification theory in order to reduce the question of resolution of singularities to the study of Galois extensions of degree $p$ and the search for suitable normal forms of Artin-Schreier-like minimal polynomials.

- Model theory of valued fields. If $\mathcal{L}$ is an elementary language and $\mathcal{A} \subset \mathcal{B}$ are $\mathcal{L}$-structures, then we will say that $\mathcal{A}$ is existentially closed in $\mathcal{B}$ and write $\mathcal{A} \prec_{\exists} \mathcal{B}$ if every existential sentence with parameters from $\mathcal{A}$ that holds in $\mathcal{B}$ also holds in $\mathcal{A}$. When we talk of fields, then we use the language of rings or fields. When we talk of valued fields, we augment this language by a unary predicate for the valuation ring or a binary predicate for valuation divisibility. For ordered abelian groups, we use the language of groups augmented by a binary predicate for the ordering. For the meaning of "existentially closed in" in the setting of valued fields and of ordered abelian groups, see $[\mathrm{K}-\mathrm{P}$. In [K7] we use Theorem 1.1 to prove the following Ax-Kochen-Ershov Principle:

Theorem 1.5. Take a henselian defectless valued field $(K, v)$ and an extension $(L \mid K, v)$ of finite transcendence degree without transcendence defect. If $v K$ is existentially closed in $v L$ and $\bar{K}$ is existentially closed in $\bar{L}$, then $(K, v)$ is existentially closed in $(L, v)$.

This in turn is used to prove Ax-Kochen-Ershov Principles and further model theoretic results for tame valued fields.

\section{Preliminaries}

2.1. Facts from general valuation theory. We will use the following facts throughout the paper, often without citing. The first lemma is a consequence of the fundamental inequality (1.1).

Lemma 2.1. Let $(L \mid K, v)$ be an algebraic extension of valued fields. Then $v L / v K$ is a torsion group and the extension $L v \mid K v$ of residue fields is algebraic. If $L \mid K$ is finite, then so are $v L / v K$ and $L v \mid K v$. If $v$ is trivial on $K$ (i.e., $v K=\{0\}$ ), then $v$ is trivial on $L$.

For each extension of the valuation $v$ from $K$ to its algebraic closure $\tilde{K}$, the value group of $\tilde{K}$ is divisible and the residue field of $\tilde{K}$ is algebraically closed.

An extension $(L \mid K, v)$ is called immediate if the canonical embeddings of $v K$ in $v L$ and of $K v$ in $L v$ are onto. For the next results and general background in valuation theory, we refer the reader to $[\mathrm{En},[\mathrm{R}, \mathrm{W}], \mathrm{Z}-\mathrm{S}]$.

Lemma 2.2. The henselization $K^{h}$ of a valued field $(K, v)$ (which is unique up to a valuation preserving isomorphism over $K$ ) is an immediate separable-algebraic extension and has the following universal property: if $\left(L, v^{\prime}\right)$ is an arbitrary henselian extension field of $(K, v)$, then there is a unique valuation preserving embedding of $\left(K^{h}, v\right)$ in $\left(L, v^{\prime}\right)$ over $K$. Thus, a henselization of $(K, v)$ can be chosen in every henselian valued extension field of $(K, v)$. 
Lemma 2.3. An algebraic extension of a henselian valued field, equipped with the unique extension of the valuation, is again henselian.

If $L \mid K$ is algebraic, then $\left(L . K^{h}, v\right)$ is the henselization of $(L, v)$ (with respect to the unique extension of $v$ from $K^{h}$ to $\left.\tilde{K}\right)$.

Take any valued field $(K, w)$ and a valuation $\bar{w}$ on the residue field $K w$. Then the composition $w \circ \bar{w}$ is the valuation whose valuation ring is the subring of the valuation ring of $w$ consisting of all elements whose $w$-residues lie in the valuation ring of $\bar{w}$. (Note that we identify equivalent valuations.) While $w \circ \bar{w}$ does not actually mean the composition of $w$ and $\bar{w}$ as mappings, this notation is used because, up to equivalence, the place associated with $w \circ \bar{w}$ is indeed the composition of the places associated with $w$ and $\bar{w}$.

For a valued field $(K, v)$, every convex subgroup $\Gamma$ of $v K$ gives rise to a valuation $v_{\Gamma}$ such that $v_{\Gamma} K$ is isomorphic to $v K / \Gamma$ and the valuation ring of $v$ is contained in that of $v_{\Gamma}$. Then $v$ induces a valuation $\bar{v}_{\Gamma}$ on $K v_{\Gamma}$ (whose valuation ring is the image of the valuation ring of $v$ under the place associated with $\left.v_{\Gamma}\right)$, such that $v=v_{\Gamma} \circ \bar{v}_{\Gamma}$. The value group $\bar{v}_{\Gamma}\left(K v_{\Gamma}\right)$ of $\bar{v}_{\Gamma}$ can be identified with $\Gamma$ and its residue field $\left(K v_{\Gamma}\right) \bar{v}_{\Gamma}$ with $K v$.

The rank of a valued field $(K, v)$ is the order type of the chain of non-trivial convex subgroups of its value group $v K$. It has rank 1 (i.e., its only convex subgroups are $\{0\}$ and $v K$ ) if and only if $v K$ is archimedean, that is, embeddable in the ordered additive group of the reals. If $(K, v)$ has finite rank $n$, then $v$ is the composition $v=v_{1} \circ \ldots \circ v_{n}$ of $n$ many rank 1 valuations. The following lemma does not in general hold for valuations of rank $>1$ :

Lemma 2.4. If $(K, v)$ is a valued field of rank 1 , then $K$ is dense in its henselization. In particular, the completion of $(K, v)$ is henselian.

For the easy proof of the following lemma, see [B], chapter VI, $\S 10.3$, Theorem 1.

Lemma 2.5. Let $(L \mid K, v)$ be an extension of valued fields. Take elements $x_{i}, y_{j} \in$ $L, i \in I, j \in J$, such that the values $v x_{i}, i \in I$, are rationally independent over $v K$, and the residues $y_{j} v, j \in J$, are algebraically independent over $K v$. Then the elements $x_{i}, y_{j}, i \in I, j \in J$, are algebraically independent over $K$.

Moreover, if we write

$$
f=\sum_{k} c_{k} \prod_{i \in I} x_{i}^{\mu_{k, i}} \prod_{j \in J} y_{j}^{\nu_{k, j}} \in K\left[x_{i}, y_{j} \mid i \in I, j \in J\right]
$$

in such a way that for every $k \neq \ell$ there is some $i$ s.t. $\mu_{k, i} \neq \mu_{\ell, i}$ or some $j$ s.t. $\nu_{k, j} \neq \nu_{\ell, j}$, then

$$
v f=\min _{k} v c_{k} \prod_{i \in I} x_{i}^{\mu_{k, i}} \prod_{j \in J} y_{j}^{\nu_{k, j}}=\min _{k} v c_{k}+\sum_{i \in I} \mu_{k, i} v x_{i} .
$$

That is, the value of the polynomial $f$ is equal to the least of the values of its monomials. In particular, this implies

$$
\begin{aligned}
v K\left(x_{i}, y_{j} \mid i \in I, j \in J\right) & =v K \oplus \bigoplus_{i \in I} \mathbb{Z} v x_{i}, \\
K\left(x_{i}, y_{j} \mid i \in I, j \in J\right) v & =K v\left(y_{j} v \mid j \in J\right) .
\end{aligned}
$$

Moreover, the valuation $v$ on $K\left(x_{i}, y_{j} \mid i \in I, j \in J\right)$ is uniquely determined by its restriction to $K$, the values $v x_{i}$ and the residues $y_{j} v$. 
Corollary 2.6. Take a valued function field $(F \mid K, v)$ without transcendence defect and set $r=\operatorname{rr} v F / v K$ and $s=\operatorname{trdeg} \bar{F} \mid \bar{K}$. Choose elements $x_{1}, \ldots, x_{r}$, $y_{1}, \ldots, y_{s} \in F$ such that the values $v x_{1}, \ldots, v x_{r}$ are rationally independent over $v K$ and the residues $\bar{y}_{1}, \ldots, \bar{y}_{s}$ are algebraically independent over $\bar{K}$. Then $\mathcal{T}=$ $\left\{x_{1}, \ldots, x_{r}, y_{1}, \ldots, y_{s}\right\}$ is a standard valuation transcendence basis $(F \mid K, v)$. Moreover, $v F / v K$ and the extension $\bar{F} \mid \bar{K}$ are finitely generated.

Proof. By the previous theorem, the elements $x_{1}, \ldots, x_{r}, y_{1}, \ldots, y_{s}$ are algebraically independent over $K$. Since $\operatorname{trdeg} F \mid K=r+s$ by assumption, these elements form a basis and hence a standard valuation transcendence basis of $(F \mid K, v)$.

It follows that the extension $F \mid K(\mathcal{T})$ is finite. By the fundamental inequality (1.1), this yields that $v F / v K(\mathcal{T})$ and $\bar{F} \mid \overline{K(\mathcal{T})}$ are finite. Since $v K(\mathcal{T}) / v K$ and $\overline{K(\mathcal{T})} \mid \bar{K}$ are already finitely generated by the foregoing lemma, it follows that $v F / v K$ and $\bar{F} \mid \bar{K}$ are also finitely generated.

Since an infinite number of distinct convex subgroups in an ordered abelian group give rise to an infinite number of rationally independent elements in that group, the following is also a consequence of Lemma 2.5.

Corollary 2.7. Every valued field of finite transcendence degree over its prime field has finite rank.

Lemma 2.8. If $(F \mid K, v)$ is a valued function field without transcendence defect and $v=w \circ \bar{w}$, then $(F \mid K, w)$ and $(F w \mid K w, \bar{w})$ are valued function fields without transcendence defect.

Proof. The value group $\bar{w}(F w)$ can be considered (in a canonical way) a subgroup of $v F$, and then $w F$ is isomorphic to $v F / \bar{w}(F w)$; the same holds for $K$ in the place of $F$. From this it follows that $\operatorname{rr} v F / v K=\operatorname{rr} w F / w K+\operatorname{rr} \bar{w}(F w) / \bar{w}(K w)$. Hence,

$$
\begin{aligned}
\operatorname{trdeg} F \mid K & =\operatorname{rr} v F / v K+\operatorname{trdeg} F v \mid K v \\
& =\operatorname{rr} w F / w K+\operatorname{rr} \bar{w}(F w) / \bar{w}(K w)+\operatorname{trdeg}(F w) \bar{w} \mid(K w) \bar{w} \\
& \leq \operatorname{rr} w F / w K+\operatorname{trdeg} F w|K w \leq \operatorname{trdeg} F| K,
\end{aligned}
$$

and we find that equality must hold everywhere. The last equality then shows that $(F \mid K, w)$ is without transcendence defect, which by Corollary 2.6 implies that $F w \mid K w$ is a function field. We also obtain that

$$
\operatorname{rr} \bar{w}(F w) / \bar{w}(K w)+\operatorname{trdeg}(F w) \bar{w}|(K w) \bar{w}=\operatorname{trdeg} F w| K w,
$$

which shows that $(F w \mid K w, \bar{w})$ is without transcendence defect.

2.2. $p$-th roots of 1-units. A well known application of Hensel's Lemma shows that in every henselian field, each 1-unit (an element of the form $1+b$ with $v b>0$ ) is an $n$-th power for every $n$ not divisible by the residue characteristic $p$. If the latter is not the case, then one considers the "level" of the 1-unit. For our purposes, we need a more precise result for a 1-unit to have a $p$-th root in a henselian field of mixed characteristic.

Throughout this paper, we will take $C$ to be an element in the algebraic closure of $\mathbb{Q}$ such that $C^{p-1}=-p$, where $p>0$ is a prime. Take a henselian field $(K, v)$ of characteristic 0 and residue characteristic $p$. Extend the valuation $v$ to $\tilde{K}$. Note that

$$
C^{p}=-p C \quad \text { and } \quad v C=\frac{1}{p-1} v p>0 .
$$


Consider the polynomial

$$
X^{p}-(1+b)
$$

with $b \in K$. Performing the transformation

$$
X=C Y+1
$$

dividing by $C^{p}$ and using the fact that $C^{p}=-p C$, we obtain the polynomial

$$
f(Y)=Y^{p}+g(Y)-Y-\frac{b}{C^{p}}
$$

with

$$
g(Y)=\sum_{i=2}^{p-1}\left(\begin{array}{c}
p \\
i
\end{array}\right) C^{i-p} Y^{i},
$$

a polynomial with coefficients in $K(C)$ of value $>0$.

Lemma 2.9. Take $(K, v)$ and $C$ as above. Then $K$ contains $C$ if and only if it contains all $p$-th roots of unity.

Proof. Since char $\bar{K}=p$, the restriction of $v$ to $\mathbb{Q} \subset K$ is the $p$-adic valuation. Since $(K, v)$ is henselian, it contains $\left(\mathbb{Q}^{h}, v_{p}\right)$. Let $\eta \neq 1$ be a $p$-th root of unity. It suffices to show that $\mathbb{Q}^{h}(\eta)=\mathbb{Q}^{h}(C)$. Applying the transformation (2.3) to the polynomial (2.2) with $b=0$, we obtain the polynomial $f(Y)=Y^{p}+g(Y)-Y \in$ $\mathbb{Q}(C)[Y]$ which splits over $\mathbb{Q}^{h}$ by Hensel's Lemma because $\bar{f}(Y)=Y^{p}-Y$ splits over $\mathbb{F}_{p}$. Since the non-zero roots of $f$ have non-zero residue and thus value zero, $v(\eta-1)=v C=v p /(p-1)$. We find that $\left(v \mathbb{Q}^{h}(\eta): v \mathbb{Q}^{h}\right) \geq p-1$. Consequently,

$$
\left[\mathbb{Q}^{h}(\eta): \mathbb{Q}^{h}\right] \geq p-1 \geq\left[\mathbb{Q}^{h}(C): \mathbb{Q}^{h}\right] \geq\left[\mathbb{Q}^{h}(\eta): \mathbb{Q}^{h}\right],
$$

showing that equality holds everywhere and that $\mathbb{Q}^{h}(\eta)=\mathbb{Q}^{h}(C)$.

Lemma 2.10. Let $(K, v)$ be a henselian field containing all $p$-th roots of unity. Then

$$
v b>\frac{p}{p-1} v p \Rightarrow 1+b \in\left(K^{\times}\right)^{p}
$$

for all $b \in K$. In other words, every 1 -unit of level $>\frac{p}{p-1}$ vp has a $p$-th root in $K$.

Proof. Consider the polynomial (2.4), derived from (2.2). If $v b>\frac{p}{p-1} v p=v C^{p}$, then $\bar{f}(Y)=Y^{p}-Y$, which splits over $\bar{K}$. By Hensel's Lemma, this implies that $f(Y)$ splits over $K$. Via the transformation (2.3), it follows that $1+b$ has a $p$-th root in $K$.

Corollary 2.11. Let $(K, v)$ be a henselian field containing all $p$-th roots of unity. Take any 1 -units $1+b$ and $1+c$ in $K$. Then:

a) $1+b \in(1+b+c) \cdot\left(K^{\times}\right)^{p} \quad$ if $v c>\frac{p}{p-1} v p$.

b) $1+b \in(1+b+c) \cdot\left(K^{\times}\right)^{p} \quad$ if $1+c \in\left(K^{\times}\right)^{p}$ and $v b c>\frac{p}{p-1} v p$.

c) $1+c^{p}+p c \in\left(K^{\times}\right)^{p} \quad$ if $v c^{p}>v p$.

d) $1+b-p c \in\left(1+b+c^{p}\right) \cdot\left(K^{\times}\right)^{p} \quad$ if $v b \geq \frac{1}{p-1} v p$ and $v c^{p}>v p$.

Proof. a): $1+b \in(1+b+c)\left(K^{\times}\right)^{p}$ is true if the quotient

$$
\frac{1+b+c}{1+b}=1+\frac{c}{1+b}
$$


is an element of $\left(K^{\times}\right)^{p}$. By hypothesis we have $v b>0$ and thus $v \frac{c}{1+b}=v c$. Now our assertion follows from Lemma 2.10.

b): An application of part a) shows that

$$
(1+b+c) \in(1+b)(1+c) \cdot\left(K^{\times}\right)^{p} \text { if } v b c>\frac{p}{p-1} v p .
$$

The assertion of $\mathrm{b}$ ) is an immediate consequence of this.

c): If $v c^{p}>v p$, then for every $i=2, \ldots, p-1$ we have

$$
v\left(\begin{array}{c}
p \\
i
\end{array}\right) c^{i} \geq v p+2 v c>\frac{p+2}{p} v p \geq \frac{p}{p-1} v p ;
$$

note that the last inequality holds for every $p \geq 2$. This, together with assertion a), yields

$$
1+c^{p}+p c \in\left(1+c^{p}+p c+\sum_{i=2}^{p-1}\left(\begin{array}{c}
p \\
i
\end{array}\right) c^{i}\right)\left(K^{\times}\right)^{p}=(1+c)^{p}\left(K^{\times}\right)^{p}=\left(K^{\times}\right)^{p} .
$$

d): In view of part c), the assertion follows from part b), where $b$ is replaced by $b-p c$ and $c$ is replaced by $c^{p}+p c$. Note that b) can be applied since

$$
v(b-p c)\left(c^{p}+p c\right)>\frac{1}{p-1} v p+v p=\frac{p}{p-1} v p .
$$

2.3. The defect. Assume that $(L \mid K, v)$ is a finite extension and the extension of $v$ from $K$ to $L$ is unique. Then the Lemma of Ostrowski says that

$$
[L: K]=(v L: v K) \cdot[L v: K v] \cdot p^{\nu} \quad \text { with } \nu \geq 0,
$$

where $p$ is the characteristic exponent of $K v$, that is, $p=$ char $K v$ if this is positive, and $p=1$ otherwise (cf. $[\mathrm{En},[\underline{\mathrm{R}})$ ). The factor

$$
\mathrm{d}(L \mid K, v):=p^{\nu}=\frac{[L: K]}{(v L: v K)[L v: K v]}
$$

is called the defect of the extension $(L \mid K, v)$. If $\nu>0$, then we talk of a nontrivial defect. If $[L: K]=p$, then $(L \mid K, v)$ has a non-trivial defect if and only if it is immediate, i.e., $(v L: v K)=1$ and $[L v: K v]=1$. If $\mathrm{d}(L \mid K, v)=1$, then we call $(L \mid K, v)$ a defectless extension. Note that $(L \mid K, v)$ is always defectless if char $K v=0$. Therefore,

Corollary 2.12. Every valued field $(K, v)$ with char $K v=0$ is a defectless field.

The following lemma shows that the defect is multiplicative. This is a consequence of the multiplicativity of the degree of field extensions and of ramification index and inertia degree. We leave the straightforward proof to the reader.

Lemma 2.13. Fix an extension of a valuation $v$ from $K$ to its algebraic closure. If $L \mid K$ and $M \mid L$ finite extensions and the extension of $v$ from $K$ to $M$ is unique, then

$$
\mathrm{d}(M \mid K, v)=\mathrm{d}(M \mid L, v) \cdot \mathrm{d}(L \mid K, v) .
$$

In particular, $(M \mid K, v)$ is defectless if and only if $(M \mid L, v)$ and $(L \mid K, v)$ are defectless. 
Theorem 2.14. Take a valued field $(K, v)$ and fix an extension of $v$ to $\tilde{K}$. Then $(K, v)$ is defectless if and only if its henselization $(K, v)^{h}$ in $(\tilde{K}, v)$ is defectless. The same holds for "separably defectless" and "inseparably defectless" in place of "defectless".

Proof. For "separably defectless", our assertion follows directly from En, Theorem (18.2). The proof of that theorem can easily be adapted to prove the assertion for "inseparably defectless" and "defectless". See [K6] for more details.

Since a henselian field has a unique extension of the valuation to every algebraic extension field, we obtain:

Corollary 2.15. A valued field $(K, v)$ is defectless if and only if $\mathrm{d}\left(L \mid K^{h}, v\right)=1$ for every finite extension $L \mid K^{h}$.

Using this corollary together with Lemma 2.13 one easily shows:

Corollary 2.16. Every finite extension of a defectless field is again a defectless field.

Lemma 2.17. Let $(K, v)$ be a valued field with $v=w \circ \bar{w}$. If $(K, w)$ and $(K w, \bar{w})$ are defectless fields, then so is $(K, v)$.

Proof. Take a finite extension $L \mid K$; we wish to show that equality holds in (1.1). Let $w_{1}, \ldots, w_{\mathrm{g}_{w}}$ be all extensions of $w$ from $K$ to $L$, and set $\mathrm{e}_{i}^{w}=\left(w_{i} L: w K\right)$ and $\mathrm{f}_{i}^{w}=\left[L w_{i}: K w\right]$ for $1 \leq i \leq \mathrm{g}_{w}$. Further, for $1 \leq i \leq \mathrm{g}_{w}$, let $\bar{w}_{i 1}, \ldots, \bar{w}_{i \mathrm{~g}_{i}}$ be all extensions of $\bar{w}$ from $K w$ to $L w_{i}$, and set $\mathrm{e}_{i j}=\left(\bar{w}_{i j}\left(L w_{i}\right): \bar{w}(K w)\right)$ and $\mathrm{f}_{i j}=\left[\left(L w_{i}\right) \bar{w}_{i j}:(K w) \bar{w}\right]=\left[L\left(w_{i} \circ \bar{w}_{i j}\right): K v\right]$ for $1 \leq j \leq \mathrm{g}_{i}$. Since $(K, w)$ is a defectless field, we have $[L: K]=\sum_{i=1}^{\mathrm{g}_{w}} \mathrm{e}_{i}^{w} \mathrm{f}_{i}^{w}$. Since $(K w, \bar{w})$ is a defectless field, we have $\mathrm{f}_{i}^{w}=\left[L w_{i}: K w\right]=\sum_{j=1}^{\mathrm{g}_{i}} \mathrm{e}_{i j} \mathrm{f}_{i j}$. Using the fact that $\mathrm{e}_{i}^{w} \mathrm{e}_{i j}=$ $\left(\left(w_{i} \circ \bar{w}_{i j}\right) L: v K\right)$, we obtain

$$
[L: K]=\sum_{i=1}^{\mathrm{g}_{w}} \mathrm{e}_{i}^{w} \sum_{j=1}^{\mathrm{g}_{i}} \mathrm{e}_{i j} \mathrm{f}_{i j}=\sum_{i=1}^{\mathrm{g}_{w}} \sum_{j=1}^{\mathrm{g}_{i}}\left(\left(w_{i} \circ \bar{w}_{i j}\right) L: v K\right)\left[L\left(w_{i} \circ \bar{w}_{i j}\right): K v\right] .
$$

As the valuations $w_{i} \circ \bar{w}_{i j}, 1 \leq i \leq \mathrm{g}_{w}, 1 \leq j \leq \mathrm{g}_{i}$, are distinct extensions of $v$ from $K$ to $L$, the fundamental inequality implies that they are in fact all extensions, and we have proved that equality holds in (1.1).

In [K6] we have proved the following:

Proposition 2.18. Let $(K, v)$ be a henselian field and $N$ an arbitrary algebraic extension of $K$ within $K^{r}$. If $L \mid K$ is a finite extension, then

$$
\mathrm{d}(L \mid K, v)=\mathrm{d}(L . N \mid N, v) .
$$

Hence, $(K, v)$ is a defectless field if and only if $(N, v)$ is a defectless field. The same holds for "separably defectless" and "inseparably defectless" in place of "defectless".

2.4. Valuation disjoint and valuation regular extensions. Let $(L \mid K, v)$ be an extension of valued fields, and let $\mathcal{B} \subset L$. Then $\mathcal{B}$ is said to be a valuation independent set in $(L \mid K, v)$ if for every $n \in \mathbb{N}$ and each choice of distinct elements $b_{1}, \ldots, b_{n} \in \mathcal{B}$ and arbitrary elements $c_{1}, \ldots, c_{n} \in K$,

$$
v \sum_{i=1}^{v} c_{i} b_{i}=\min _{1 \leq i \leq n} v c_{i} b_{i}
$$


Further, $\mathcal{B}$ is called a standard valuation independent set (in $(L \mid K, v)$ ) if it is of the form $\mathcal{B}=\left\{b_{i}^{\prime} b_{j}^{\prime \prime} \mid 1 \leq i \leq k, 1 \leq j \leq \ell\right\}$, where the values $v b_{1}^{\prime}, \ldots, v b_{k}^{\prime}$ lie in distinct cosets of $v L$ modulo $v K$, and $b_{1}^{\prime \prime}, \ldots, b_{\ell}^{\prime \prime}$ are elements of value 0 whose residues are $\bar{K}$-linearly independent. Every standard valuation independent set is a valuation independent set.

Let $(\Omega \mid K, v)$ be an extension of valued fields and $F \mid K$ and $L \mid K$ be two subextensions of $\Omega \mid K$. We say that $(F \mid K, v)$ is valuation disjoint from $(L \mid K, v)$ (in $(\Omega, v))$ if every standard valuation independent set $\mathcal{B}$ of $(F \mid K, v)$ is also a standard valuation independent set of $(L . F \mid L, v)$. (It is possible that a standard valuation independent set $\mathcal{B}$ of $(F \mid K, v)$ remains valuation independent over $(L, v)$ without remaining a standard valuation independent set of $(L . F \mid L, v)$.)

Let $G$ and $G^{\prime}$ be two subgroups of some group $\mathcal{G}$ and $H$ be a common subgroup of $G$ and $G^{\prime}$. We will say that the group extension $G \mid H$ is disjoint from the group extension $G^{\prime} \mid H$ (in $\mathcal{G}$ ) if every two elements in $G$ that belong to distinct cosets modulo $H$ will also belong to distinct cosets modulo $G^{\prime}$. This holds if and only if $G \cap G^{\prime}=H$. Hence, our notion "disjoint from" is symmetrical, like "linearly disjoint from" in the field case.

Lemma 2.19. Let $(\Omega \mid K, v)$ be an extension of valued fields and $F \mid K$ and $L \mid K$ be subextensions of $\Omega \mid K$. Then $(F \mid K, v)$ is valuation disjoint from $(L \mid K, v)$ in $(\Omega, v)$ if and only if

1) $v F \mid v K$ is disjoint from $v L \mid v K$ in $v \Omega$, and

2) $\bar{F} \mid \bar{K}$ is linearly disjoint from $\bar{L} \mid \bar{K}$ in $\bar{\Omega}$.

Consequently, the notion of "valuation disjoint" is symmetrical.

Proof. Let $\mathcal{B}$ be a standard valuation independent set in $(F \mid K, v)$ of the form as given in the definition. By condition 1$)$, the values $v b_{1}^{\prime}, \ldots, v b_{k}^{\prime}$ also lie in distinct cosets of $v(L . F)$ modulo $v L$. By condition 2), the residues $b_{1}^{\prime \prime}, \ldots, v b_{\ell}^{\prime \prime}$ remain $\bar{L}$-linearly independent. Hence, $\mathcal{B}$ is a standard valuation independent set in $(L . F \mid L, v)$.

For the converse, assume that condition 1) or 2) is not satisfied. If 1) is not satisfied, then there are two elements $b, b^{\prime} \in F$ whose values belong to distinct cosets modulo $v K$ but to the same coset modulo $v L$. Hence, $\left\{b, b^{\prime}\right\}$ is a standard valuation independent set in $(F \mid K, v)$, but not in $(L . F \mid L, v)$. If 2$)$ is not satisfied, then there are elements $b_{1}, \ldots, b_{n} \in F$ of value 0 whose residues are $\bar{K}$-linearly independent but not $\bar{L}$-linearly independent. Then $\left\{b_{1}, \ldots, b_{n}\right\}$ is a standard valuation independent set in $(F \mid K, v)$, but not in $(L . F \mid L, v)$.

Recall that an extension $F \mid K$ is called regular if it is linearly disjoint from $\tilde{K} \mid K$, or equivalently, if it is separable and $K$ is relatively algebraically closed in $F$. An extension $(F \mid K, v)$ of valued fields will be called valuation regular if it is valuation disjoint from $(\tilde{K} \mid K, v)$ in $(\tilde{F}, v)$ for some extension of $v$ from $F$ to $\tilde{F}$. Using the fact that $v \tilde{K}$ is the divisible hull of $K$ and $\bar{K}$ is the algebraic closure of $\bar{K}$ (Lemma 2.1), one deduces:

Lemma 2.20. An extension $(F \mid K, v)$ is valuation regular if and only if

1) $v F / v K$ is torsion free,

2) $\bar{F} \mid \bar{K}$ is regular.

Consequently, $(F \mid K, v)$ is valuation regular if and only if it is valuation disjoint 
from $(\tilde{K} \mid K, v)$ for every extension of $v$ from $F$ to $\tilde{F}$. Every valued field extension of an algebraically closed valued field is valuation regular.

Since the henselization of a valued field is an immediate extension, this lemma yields:

Corollary 2.21. If $(F \mid K, v)$ is valuation regular, then $\left(F^{h} \mid K^{h}, v\right)$ is also valuation regular.

Important examples of valuation regular extensions are the valued field extensions which are generated by standard algebraically valuation independent sets. Indeed, it follows from Lemma 2.5 that they satisfy the conditions of the above lemma. Also using Lemma 2.19, we obtain:

Lemma 2.22. Let $(\Omega \mid K, v)$ be an extension of valued fields containing a standard algebraically valuation independent set $\mathcal{T}$. Then $(K(\mathcal{T}) \mid K, v)$ is a valuation regular extension.

Lemma 2.23. Assume that $(F \mid K, v)$ is a valuation regular subextension of a valued field extension $(\Omega \mid K, v)$. If $\mathcal{T}$ is a standard algebraically valuation independent set in $(\Omega \mid F, v)$, then $(F(\mathcal{T}) \mid K(\mathcal{T}), v)$ is also a valuation regular extension.

Proof. We write $\mathcal{T}=\left\{x_{i}, y_{j} \mid i \in I, j \in J\right\}$, where the values $v x_{i}, i \in I$, are rationally independent over $v F$, and the residues $\bar{y}_{i}, j \in J$, are algebraically independent over $\bar{F}$. Since $(F \mid K, v)$ is valuation regular, Lemma 2.20 shows that $v F / v K$ is torsion free and that $\bar{F} \mid \bar{K}$ is regular. The former implies that $v F \oplus \bigoplus_{i \in I} \mathbb{Z} v x_{i}$ is also torsion free modulo $v K \oplus \bigoplus_{i \in I} \mathbb{Z} v x_{i}$. The latter implies that the extension $\bar{F}\left(\bar{y}_{j} \mid j \in J\right) \mid \bar{K}\left(\bar{y}_{j} \mid j \in J\right)$ is also regular (this follows from the fact that the elements $\bar{y}_{j}$ are algebraically independent over $\bar{F}$ ). Again by Lemma 2.20 , $(F(\mathcal{T}) \mid K(\mathcal{T}), v)$ is a valuation regular extension.

Here is the reason why we consider valuation disjoint extensions:

Proposition 2.24. Take an extension $(F \mid K, v)$ of henselian fields and a finite algebraic extension $(L \mid K, v)$, valuation disjoint from $(F \mid K, v)$ (in $(\tilde{F}, v))$. Then

$$
\mathrm{d}(L \mid K, v) \geq \mathrm{d}(L . F \mid F, v) .
$$

Proof. Since $v L \mid v K$ is disjoint from $v F \mid v K$ and $\bar{L} \mid \bar{K}$ is linearly disjoint from $\bar{F} \mid \bar{K}$, we find that

$$
\left\{\begin{aligned}
(v(L . F): v F) & \geq(v L+v F: v F)=(v L: v K) \\
{[\overline{L . F}: \bar{F}] } & \geq[\bar{L} \cdot \bar{F}: \bar{F}]=[\bar{L}: \bar{K}]
\end{aligned}\right.
$$

Now inequality (2.8) follows from this, together with $\left[(L . F)^{h}: F^{h}\right] \leq\left[L^{h}: K^{h}\right]$.

Corollary 2.25. Take a valuation regular extension $(F \mid K, v)$, fix an extension of $v$ from $F$ to $\tilde{F}$, and assume that $(K, v)$ and $(\tilde{K} . F, v)$ are defectless fields. Then $(F, v)$ is also a defectless field.

Proof. From Theorem 2.14 we know that $\left(K^{h}, v\right)$ and $\left((\tilde{K} . F)^{h}, v\right)$ are defectless fields and that it suffices to prove that $\left(F^{h}, v\right)$ is a defectless field. We will prove that every finite subextension $E \mid F^{h}$ of $(\tilde{K} . F)^{h} \mid F^{h}$ is defectless. Since $\left((\tilde{K} . F)^{h}, v\right)$ 
is a defectless field, it will then follow from Lemma 2.3 of $\left[\mathrm{K} 6\right.$ that $\left(F^{h}, v\right)$ is a defectless field.

Since $(\tilde{K} . F)^{h}=\tilde{K} . F^{h}$ by Lemma 2.3. there is a finite extension $L \mid K^{h}$ such that $E \subseteq L . F^{h}$. Since $\left(K^{h}, v\right)$ is a defectless field, we know that $\mathrm{d}\left(L \mid K^{h}, v\right)=1$. Since $(F \mid K, v)$ is assumed to be valuation regular, the same holds for $\left(F^{h} \mid K^{h}, v\right)$ by Corollary 2.21. Thus it follows from Lemma 2.24 that $\mathrm{d}\left(L \cdot F^{h} \mid F^{h}, v\right) \leq \mathrm{d}\left(L \mid K^{h}, v\right)=1$. Using Lemma 2.13 we conclude that $\mathrm{d}\left(E \mid F^{h}, v\right)=1$.

2.5. Henselized function fields. Theorem 2.14 shows that a valued field is defectless if and only if its henselization is defectless. So instead of working with a valued function field, we will rather analyze its henselization. Such a henselization will be called a henselized function field. Every finite extension of a henselized function field is again a henselized function field. Further, a henselized function field $(F \mid K, v)$ will be called henselized rational with generator $x$ if $F=K(x)^{h}$. We will say that $(F \mid K, v)$ is henselized inertially generated with generator $x$ if $(F, v)$ is a finite unramified extension of a henselized rational function field with generator $x$.

We note that $(F \mid K, v)$ is henselized inertially generated with generator $x$ if and only if there exists some $y \in F$ such that $F=K(x)^{h}(y), v y=0$ and $\overline{K(x)}(\bar{y}) \mid \overline{K(x)}$ is a finite separable extension of degree

$$
[\overline{K(x)}(\bar{y}): \overline{K(x)}]=[K(x, y): K(x)]=\left[K(x)^{h}(y): K(x)^{h}\right] .
$$

Indeed, if the latter holds, then $F \mid K(x)^{h}$ is unramified. Conversely, if $F \mid K(x)^{h}$ is unramified, then $\bar{F} \mid \overline{K(x)}$ is a finite separable extension, of degree $\left[F: K(x)^{h}\right]$, and we can choose some $\zeta \in \bar{F}$ such that $\bar{F}=\overline{K(x)}(\zeta)$. Take a monic polynomial $f$ with coefficients in the valuation ring of $K(x)$ and such that $\bar{f}$ is the minimal polynomial of $\zeta$ over $\overline{K(x)}$. By Hensel's Lemma, $f$ has a root $y \in F$ such that $\bar{y}=\zeta$. Since $\operatorname{deg}(f)=\operatorname{deg}(\bar{f})$, we obtain the first equation in (2.10). Consequently,

$$
[\overline{K(x)}(\bar{y}): \overline{K(x)}]=[K(x, y): K(x)] \geq\left[K(x)^{h}(y): K(x)^{h}\right] \geq[\overline{K(x)}(\bar{y}): \overline{K(x)}] .
$$

Hence, equality holds everywhere, showing that $F=K(x)^{h}(y)$ and that the second equation in (2.10) holds. Note that $v F=v K(x)$ by Lemma 2.5.

Take a henselized inertially generated function field $(F \mid K, v)$ of transcendence degree 1 without transcendence defect. Then either $\operatorname{rr} v F / v K=1$ and there is a value-transcendental element $x \in F$, i.e., its value $v x$ is rationally independent over $v K$, or $\operatorname{trdeg} \bar{F} \mid \bar{K}=1$ and there is a residue-transcendental element $x \in$ $F$, i.e., $v x=0$ and the residue $\bar{x}$ is transcendental over $\bar{K}$. In both cases, $x$ is transcendental over $K$ by Lemma 2.5, hence $F \mid K(x)$ is finite.

Assume in addition that the element $x$ is a generator for the henselized inertially generated function field $(F \mid K, v)$. Then in the first case, we call $x$ a valuetranscendental generator, and in the second case a residue-transcendental generator for $(F \mid K, v)$. In both cases, we also call $x$ a valuation-transcendental generator. In the first (the "value-transcendental") case, $\bar{F} \mid \bar{K}$ is finite, and in the second (the "residue-transcendental") case, $v F / v K$ is a finite torsion group (cf. Corollary 2.6).

If in addition $K$ is algebraically closed, then by Lemma 2.1, $\bar{F}=\bar{K}$ is algebraically closed and $v K$ is divisible in the value-transcendental case, and $v F=v K$ is divisible and $\bar{K}$ algebraically closed in the residue-transcendental case. We see 
that a henselized rational function field of transcendence degree 1 with a valuetranscendental generator over an algebraically closed field does not admit proper unramified extensions. Hence:

Lemma 2.26. Every henselized inertially generated function field of transcendence degree 1 with a value-transcendental generator over an algebraically closed field is a henselized rational function field.

Lemma 2.27. Take an algebraically closed field $K$ and a finite extension $(E \mid F, v)$ within $F^{r}$. If $(F \mid K, v)$ is a henselized rational function field with a value-transcendental generator, then so is $(E \mid K, v)$. If $(F \mid K, v)$ is a henselized inertially generated function field with a residue-transcendental generator, then so is $(E \mid K, v)$, with the same generator.

Proof. Case I): $(F \mid K, v)$ has a value-transcendental generator $x$, so $F=K(x)^{h}$. Since $v F=v K(x)=v K \oplus \mathbb{Z} v x, v K$ is divisible and $v E \mid v F$ is finite, we have that $v E=v K \oplus \mathbb{Z} \frac{v x}{n}$ for some $n \geq 1$. Since $E \subset F^{r}, n$ is equal to $[E: F]$ and prime to char $\bar{K}$. As $\bar{F}=\bar{K}$ is algebraically closed and $\bar{E} \mid \bar{F}$ is finite, we find that $\bar{E}=\bar{F}$. Therefore, Hensel's Lemma can be used to find an element $y$ in the henselian field $E$ such that $y^{n}=c x$ for some $c \in F$ of value 0 . We have that $v y=\frac{v x}{n}, K(x)^{h} \subset K(y)^{h}, \overline{K(y)}=\overline{K(x)}$ and

$$
\begin{aligned}
{\left[E: K(x)^{h}\right] } & \geq\left[K(y)^{h}: K(x)^{h}\right] \geq(v K(y): v K(x)) \cdot[\overline{K(y)}: \overline{K(x)}] \\
& =(v K(y): v K(x)) \geq n=\left[E: K(x)^{h}\right] .
\end{aligned}
$$

Hence, equality holds everywhere, so $E=K(y)^{h}$. As $v y$ is rationally independent over $v K,(E \mid K, v)$ is a henselized rational function field with a value-transcendental generator $y$.

Case II): $(F \mid K, v)$ has a residue-transcendental generator $x$. Then $v F=v K$ is divisible, and since $v E / v F$ is finite, $v E=v F$. Hence $(E \mid F, v)$ is unramified and $(E \mid K, v)$ is again a henselized inertially generated function field with a generator $x$.

\section{INSEPARABLY DEFECTLESS FIELDS}

We shall prove the "inseparably defectless" version of the Generalized Stability Theorem:

Proposition 3.1. Let $(F \mid K, v)$ be a valued function field without transcendence defect. If $(K, v)$ is an inseparably defectless field, then so is $(F, v)$.

Proof. We choose a standard valuation transcendence basis $\mathcal{T}$ of $(F \mid K, v)$ as in Corollary 2.6. Then $F \mid K(\mathcal{T})$ is a finite extension. By Lemma 4.15 of [K6], every finite extension of an inseparably defectless field is again an inseparably defectless field. Hence it suffices to prove that $(K(\mathcal{T}), v)$ is an inseparably defectless field.

Every finite purely inseparable extension $L$ of $K(\mathcal{T})$ is contained in an extension $E=K^{\prime}\left(\mathcal{T}^{1 / p^{m}}\right)=K^{\prime}\left(t^{1 / p^{m}} \mid t \in \mathcal{T}\right)$ for a suitable $m \in \mathbb{N}$ and some finite purely inseparable extension $K^{\prime}$ of $K$. Since $K^{\prime} \mid K$ is algebraic, we know from Lemma 2.1 that $v K^{\prime} / v K$ is a torsion group and $\overline{K^{\prime}} \mid \bar{K}$ is algebraic. Consequently, the values $v x_{i}^{1 / p^{m}}=\frac{v x_{i}}{p^{m}}, 1 \leq i \leq r$, are still rationally independent over $v K^{\prime}$, and the residues $\overline{y^{1 / p^{m}}}{ }_{i}=\bar{y}_{i}^{1 / p^{m}}, 1 \leq j \leq s$, are still algebraically independent over $\overline{K^{\prime}}$. 
This proves that $\mathcal{T}^{1 / p^{m}}$ is a standard valuation transcendence basis of $\left(E \mid K^{\prime}, v\right)$. Now Lemma 2.5 shows that

$$
v E=v K^{\prime} \oplus \mathbb{Z} v x_{1}^{1 / p^{m}} \oplus \ldots \oplus \mathbb{Z} v x_{r}^{1 / p^{m}}=v K^{\prime} \oplus \mathbb{Z} \frac{v x_{1}}{p^{m}} \oplus \ldots \oplus \mathbb{Z} \frac{v x_{r}}{p^{m}}
$$

and that

$$
\bar{E}=\overline{K^{\prime}}\left(\overline{y_{1}^{1 / p^{m}}}, \ldots, \overline{y_{s}^{1 / p^{m}}}\right)={\overline{K^{\prime}}}\left(\bar{y}^{1 / p^{m}}, \ldots,{\overline{y_{s}}}^{1 / p^{m}}\right)
$$

whence

$$
\begin{aligned}
{[E: K(\mathcal{T})] } & =\left[K^{\prime}\left(\mathcal{T}^{1 / p^{m}}\right): K^{\prime}(\mathcal{T})\right] \cdot\left[K^{\prime}(\mathcal{T}): K(\mathcal{T})\right]=p^{m(r+s)} \cdot\left[K^{\prime}: K\right] \\
& =p^{m r} \cdot p^{m s} \cdot\left(v K^{\prime}: v K\right) \cdot\left[\overline{K^{\prime}}: \bar{K}\right] \\
& =p^{m r} \cdot\left(v K^{\prime}: v K\right) \cdot p^{m s} \cdot\left[\overline{K^{\prime}}: \bar{K}\right] \\
& =(v E: v K(\mathcal{T})) \cdot[\bar{E}: \overline{K(\mathcal{T})}]
\end{aligned}
$$

since $\left(K^{\prime} \mid K, v\right)$ is defectless by hypothesis. This equation shows that $(E \mid K(\mathcal{T}), v)$ is defectless. Then by Lemma 2.13, its subextension $(L \mid K(\mathcal{T}), v)$ is also defectless.

\section{Galois extensions of Degree $p$}

In this section, we will consider the structure of Galois extensions $E \mid F$ of degree $p$ of a henselized inertially generated function field $(F \mid K, v)$ of rank 1 and of transcendence degree 1 with a valuation-transcendental generator $x$. Throughout, we will assume that

$$
\left\{\begin{array}{l}
(K, v) \text { is henselian and } p=\operatorname{char} \bar{K}>0, \\
\text { and } K \text { is closed under } p \text {-th roots. }
\end{array}\right.
$$

If char $K=p$, then the latter condition is equivalent to $K$ being perfect. If char $K=$ 0 , then it implies that $K$ contains all $p$-th roots of unity.

In Section 2.5 we have shown that if $K$ is algebraically closed, then we may assume that $F$ has the following structure:

a) The value-transcendental case: $(F \mid K, v)$ is henselized rational, so we have that

$$
F=K(x)^{h} \text { is of rank } 1 \text { and } x \text { is value-transcendental over } K \text {. }
$$

In this case, $\bar{F}=\bar{K}$ and $v F=v K \oplus \mathbb{Z} v x$.

b) The residue-transcendental case: here we have

$$
\left\{\begin{array}{l}
F=K(x)^{h}(y) \text { is of rank } 1, \text { where } x \text { is residue-transcendental over } K \\
v y=0, \\
\bar{F}=\bar{K}(\bar{x}, \bar{y}) \text { with } \bar{K} \text { relatively algebraically closed in } \bar{F}, \text { and } \\
\bar{K}(\bar{x}, \bar{y}) \mid \bar{K}(\bar{x}) \text { separable with }[\bar{K}(\bar{x}, \bar{y}): \bar{K}(\bar{x})]=[K(x, y): K(x)]
\end{array}\right.
$$

In this case, $v F=v K$.

In what follows we will not assume that $K$ is algebraically closed. Instead, we will assume (4.1) together with (4.2) or (4.3), respectively.

If char $K=p$, then the Galois extension $E \mid F$ of degree $p$ is an Artin-Schreier extension (cf. [L], Theorem 6.4). That is, the extension is of the form

$$
E=F(\vartheta), \quad \text { where } \quad a:=\vartheta^{p}-\vartheta \in F .
$$

By the additivity of the Artin-Schreier polynomial $\wp(X)=X^{p}-X$, for every $d \in F$ we have

$$
E=F(\vartheta-d), \quad \wp(\vartheta-d)=\vartheta^{p}-d^{p}-\vartheta+d=a-d^{p}+d \in a+\wp(F) .
$$


This shows that we can replace $a$ by any other element of $a+\wp(F)$ without changing the Artin-Schreier extension. Note that by Hensel's Lemma, $X^{p}-X-a$ has a root in the henselian field $F$ whenever $v a>0$. For the valuation ideal $\mathcal{M}_{F}$ of $F$, we thus have

$$
\mathcal{M}_{F} \subset \wp(F)
$$

If char $K=0$ and $K$ contains all $p$-th roots of unity, then $E \mid F$ is a Kummer extension (cf. [L], Theorem 6.2). That is, the extension is of the form

$$
E=F(\vartheta), \quad \text { where } \quad a:=\vartheta^{p} \in F .
$$

For every $d \in F^{\times}$we have

$$
E=F(\vartheta d), \quad(\vartheta d)^{p}=a d^{p} \in a\left(F^{\times}\right)^{p},
$$

showing that we can replace $a$ by any other element of $a\left(F^{\times}\right)^{p}$ without changing the extension $E \mid F$.

The main goal of this section is the proof of the following result:

Proposition 4.1. Assume that $(K, v)$ satisfies condition (4.1). Further, let (F, $v$ ) be of the form (4.2) or (4.3), and let $(E \mid F, v)$ be a Galois extension of degree $p$. Then either $(E \mid F, v)$ is defectless or there is a Galois extension $L \mid K$ of degree $p$ with non-trivial defect such that (L.E $\mid L . F, v)$ is defectless.

Corollary 4.2. Let $(F \mid K, v)$ be a henselized inertially generated function field of transcendence degree 1 and rank 1 . If $(K, v)$ is a defectless field, then every Galois extension $(E \mid F, v)$ of degree $p$ is defectless.

Remark 4.3. The normal forms we will derive will show that if we drop the condition that $K$ be closed under $p$-th roots, then the assertion of Proposition 4.1 remains true if we replace $K$ by a finite extension $K^{\prime}$ (which depends on $F$ ), $F$ by $K^{\prime} . F$ and $E$ by $K^{\prime} . E$. This extension $K^{\prime} \mid K$ can be chosen purely inseparable if char $K=p$, and can be generated by successive adjunction of $p$-th roots if char $K=0$. Indeed, it suffices to choose $K^{\prime}$ large enough to contain the finitely many coefficients that appear in the normal forms.

4.1. The value-transcendental case. We will first discuss the value-transcendental case of Proposition 4.1. We will consider the ring

$$
R=K\left[x, x^{-1}\right]
$$

which consists of all finite Laurent series

$$
\varphi(x)=\sum_{i \in I} c_{i} x^{i}, \quad c_{i} \in K, \quad I \subset \mathbb{Z} \text { finite. }
$$

Lemma 4.4. Let $F=K(x)^{h}$, where $(K(x), v)$ is of rank 1 and $x$ is value-transcendental over $K$. Then $R$ is dense in $F$. If char $K=p$, then this implies that $F=R+\wp(F)$.

Proof. Since $(K(x), v)$ is of rank 1, we know from Lemma 2.4 that it is dense in $K(x)^{h}$. Now it suffices to prove that $R$ is dense in its quotient field $K(x)$. For this, it is enough to show that for every $0 \neq \varphi(x) \in R$ and $\alpha \in v K(x)$ there is some $\tilde{\varphi}(x) \in R$ such that

$$
v\left(\frac{1}{\varphi(x)}-\tilde{\varphi}(x)\right)>\alpha
$$


Using the notation of (4.9) we have that

$$
v \varphi(x)=\min _{i \in I} v c_{i} x^{i}=v c_{k} x^{k}
$$

for a unique $k \in I$ since $x$ is value-transcendental over $K$. We write

$$
\frac{1}{\varphi(x)}=\frac{c_{k}^{-1} x^{-k}}{1-\psi(x)} \quad \text { with } \quad \psi(x)=1-c_{k}^{-1} x^{-k} \varphi(x) \in R .
$$

Since $v \psi(x)>0$ and $v K$ is archimedean by hypothesis, there is some $n \in \mathbb{N}$ such that $(n+1) v \psi(x)>\alpha+v c_{k} x^{k}$. Then by the geometric expansion, the element

$$
\tilde{\varphi}(x):=c_{k}^{-1} x^{-k} \sum_{i=0}^{n} \psi(x)^{i} \in R
$$

satisfies

$$
\begin{aligned}
v\left(\frac{1}{\varphi(x)}-\tilde{\varphi}(x)\right) & =v\left(\frac{1}{1-\psi(x)}-\sum_{i=0}^{n} \psi(x)^{i}\right)-v c_{k} x^{k} \\
& =(n+1) v \psi(x)-v c_{k} x^{k}>\alpha .
\end{aligned}
$$

We have now proved that $R$ is dense in $F$. This means that $F=R+\mathcal{M}_{F}$. If char $K=p$, then by (4.6) it follows that $F=R+\wp(F)$.

- The equal characteristic case. We deduce the following normal form, which proves the equal characteristic value-transcendental case of Proposition 4.1]

Proposition 4.5. Let $K, F$ and $E$ be as in the value-transcendental case of Proposition 4.1, and assume that $\operatorname{char} K=p$. Then either

$$
E=F(\vartheta), \text { where } \vartheta^{p}-\vartheta \in K,
$$

or

$$
E=F(\vartheta), \text { where } \vartheta^{p}-\vartheta=c_{0}+\sum_{i \in I} c_{i} x^{i}, \quad c_{i} \in K,
$$

with finite non-empty $I \subset p \mathbb{Z} \backslash \mathbb{Z}$ and such that

$$
\forall i \in I: v c_{i} x^{i}<0
$$

and $\forall i \in I: v c_{i} x^{i}<v c_{0} \leq 0$ if $c_{0} \neq 0$.

If (4.11) does not hold, then $(v E: v F)=p$ and the extension $(E \mid F, v)$ is defectless. If (4.11) holds, then for $L=K(\vartheta)$ we have that the extension (L.E|L.F, $v$ ) is trivial and hence defectless. In this case, $(E \mid F, v)$ is defectless if $(L \mid K, v)$ is defectless.

Proof. We can assume that $E \mid F$ is of the form (4.4). By Lemma 4.4 and (4.5), we can also assume that $a$ is a finite Laurent series $\varphi(x)$ of the form (4.9) and only containing summands of value $\leq 0$. Again by (4.5), we can replace a summand $c_{j p} x^{j p}$ of $\varphi(x)$ by a summand $c_{j}^{\prime} x^{j}$ with $c_{j}^{\prime}=c_{j p}^{1 / p} \in K$ (as $K$ is assumed to be perfect). After a finite repetition of this procedure we arrive at a finite Laurent series $c_{0}+\sum_{i \in I} c_{i} x^{i}$ with $I \subset \mathbb{Z} \backslash p \mathbb{Z}$. In this procedure, all summands remain of value $\leq 0$ and the original coefficient $c_{0} \in K$ remains unchanged. Note that for all $i \in I, v c_{i} x^{i}<0$ because $i \neq 0$, and thus $i v x \notin v K$.

Assume now that (4.11) does not hold. Then $I \neq \emptyset$. We may assume that $v c_{0}>v c_{i} x^{i}$ for all $i \in I$. Indeed, if this is not the case, then using (4.5) $\nu$ times, we 
can replace $c_{0}$ by $c_{0}^{1 / p^{\nu}}$. For large enough $\nu$, we obtain that $0 \geq v c_{0}^{1 / p^{\nu}}=\left(v c_{0}\right) / p^{\nu}>$ $v c_{i} x^{i}$ for all $i \in I$, because the value group $v K(x)$ is archimedean by hypothesis.

Since the elements $x^{i}, i \in \mathbb{Z}$, are valuation independent over $K$, there is $j \in I$ such that $v\left(c_{0}+\sum_{i \in I} c_{i} x^{i}\right)=v c_{j} x^{j}<0$. Therefore, we must have that $v \vartheta<0$. It follows that $v \vartheta^{p}=p v \vartheta<v \vartheta$ and consequently, $p v \vartheta=v \vartheta^{p}=v c_{j} x^{j}$ by the ultrametric triangle law. As $j \notin p \mathbb{Z}$, this value is not in $p v F$. Hence $(v E: v F) \geq p$. From the fundamental inequality it then follows that $(v E: v F)=p$.

Finally, assume that (4.11) holds. Set $L=K(\vartheta)$. Since $v F / v K$ is torsion free by assumption and $\bar{F}=\bar{K}$, we know from Lemma 2.20 that $(F \mid K, v)$ is valuation regular. Therefore, the algebraic extension $(L \mid K, v)$ is valuation disjoint from $(F \mid K, v)$ and Lemma 2.24 tells us that $\mathrm{d}(L \mid K, v) \geq \mathrm{d}(L . F \mid F, v)=\mathrm{d}(E \mid F, v)$. Hence if $(L \mid K, v)$ is defectless, then so is $(E \mid F, v)$.

- The mixed characteristic case. The following normal form proves the mixed characteristic value-transcendental case of Proposition 4.1 .

Proposition 4.6. Let $K, F$ and $E$ be as in the value-transcendental case of Proposition 4.1, and assume that char $K=0$. Then

$$
E=F(\vartheta) \text {, where } \vartheta^{p}=x^{m} u,
$$

with $m \in\{0, \ldots, p-1\}$ and $u \in K\left[x, x^{-1}\right]$ a 1-unit of the form

$$
u=1+\sum_{i \in I} c_{i} x^{i}, \quad c_{i} \in K
$$

with finite index set $I \subset \mathbb{Z} \backslash\{0\}$ and

$$
\forall i \in I: \quad 0<v c_{i} x^{i} \leq \frac{p}{p-1} v p \quad \text { and } \quad\left(i \in p \mathbb{Z} \Rightarrow v c_{i} x^{i}>v p\right) .
$$

If $v c_{i} x^{i} \geq \frac{1}{p-1}$ vp for all $i \in I$, then it may in addition be assumed that $I \subset \mathbb{Z} \backslash p \mathbb{Z}$. If $I \neq \emptyset$, then $v \sum_{i \in I} c_{i} x^{i}=\min _{i \in I} v c_{i} x^{i}$ is not divisible by $p$ in $v F$.

Further, $m \neq 0$ or $I \neq \emptyset$. In both cases, $(v E: v F)=p$.

Proof. We can assume that $E \mid F$ is of the form (4.7). Since $v F=v K \oplus \mathbb{Z} v x$ and $\bar{F}=\bar{K}$, we can write $a=c x^{k} u$, with $k \in \mathbb{Z}, c \in K$ and $u \in F$ a 1 -unit. Using (4.8) and our assumption that $K$ is closed under $p$-th roots, we may replace $a$ by $x^{m} u$, where $m=(k \bmod p)$. By Lemma 4.4 and part a) of Corollary 2.11, we may assume that

$$
u=1+\sum_{i \in I} c_{i} x^{i} \in R \quad \text { with } \quad c_{i} \in K, v c_{i} x^{i} \leq \frac{p}{p-1} v p \text { and } I \subset \mathbb{Z} \text { finite. }
$$

Since $u$ is a 1-unit, we have $v \sum c_{i} x^{i}>0$, and since the elements $x^{i}, i \in \mathbb{Z}$, are valuation independent, we find that $v c_{i} x^{i}>0$ for each $i \in I$.

Since $K$ is closed under $p$-th roots, a monomial $c_{i} x^{i}$ is a $p^{\nu}$-th power in $F$ if and only if $i \in p^{\nu} \mathbb{Z}$. Indeed, if $i \notin p^{\nu} \mathbb{Z}$, then $v\left(c_{i} x^{i}\right)^{1 / p^{\nu}}=v c_{i}^{1 / p^{\nu}}+\frac{i}{p^{\nu}} v x \notin$ $v K \oplus \mathbb{Z} v x=v F$. In a first step, we will eliminate all $p$-th powers $\neq c_{0}$ of value $\leq v p$ in the above sum. Assuming that there are any, let $\nu$ be the largest positive integer such that $p^{\nu}$-th powers $\neq c_{0}$ appear. Suppose that there are $n$ many, and write them as $z_{1}^{p^{\nu}}, \ldots, z_{n}^{p^{\nu}}$. Denote by $y$ the sum of all other summands, so that 
$u=1+y+\sum_{j=1}^{n} z_{j}^{p^{\nu}}$. Using (4.8), we replace $u$ by

$$
u^{\prime}:=\frac{u}{\left(1+\sum_{j=1}^{n} z_{j}\right)^{p^{\nu}}}=\frac{1+\sum_{j=1}^{n} z_{j}^{p^{\nu}}}{\left(1+\sum_{j=1}^{n} z_{j}\right)^{p^{\nu}}}+\frac{y}{\left(1+\sum_{j=1}^{n} z_{j}\right)^{p^{\nu}}} .
$$

Using the geometrical series expansion as in the proof of Lemma 4.4, we find that the first quotient on the right hand side is equivalent to 1 modulo $p \mathcal{M}_{F}$. The second quotient on the right hand side is equivalent modulo $p \mathcal{M}_{F}$ to a finite sum of products of the summands in $y$ with the $p^{\nu}$-th powers $z_{1}^{p^{\nu}}, \ldots, z_{n}^{p^{\nu}}$. Since the summands $\neq c_{0}$ in $y$ aren't $p^{\nu}$-th powers, the products aren't either. By the geometrical series expansion we also find that there are only finitely many summands $c_{i}^{\prime} x^{i}$ in $u^{\prime}$ of value $\leq \frac{p}{p-1} v p$. By part a) of Corollary 2.11 we can thus replace $u^{\prime}$ by the sum of these finitely many elements, which is a 1-unit. We will again call it $u$. Iterating this process until we finish with $\nu=1$, we obtain a 1-unit $u$ in which no $p$-th powers $c_{i} x^{i} \neq c_{0}$ of value $\leq v p$ appear:

$$
\begin{gathered}
u=1+\sum_{i \in I} c_{i} x^{i} \quad \text { with finite } I \subset \mathbb{Z}, 0<v c_{i} x^{i} \leq \frac{p}{p-1} v p, \\
\text { and } c_{i} x^{i} \in p \mathcal{M}_{F} \text { if } 0 \neq i \in p \mathbb{Z} .
\end{gathered}
$$

We note that

$$
\frac{u}{1+c_{0}}=\frac{1+c_{0}}{1+c_{0}}+\sum_{i \in I \backslash\{0\}} \frac{c_{i}}{1+c_{0}} x^{i}=1+\sum_{i \in I \backslash\{0\}} \frac{c_{i}}{1+c_{0}} x^{i},
$$

where the values of the summands have not changed. Since $K$ is closed under $p$-th roots, $1+c_{0}$ is a $p$-th power in $K$. We may thus replace $u$ by $\frac{u}{1+c_{0}}$ and $I$ by $I \backslash\{0\}$ so that we may assume in addition that $I \subset \mathbb{Z} \backslash\{0\}$.

Suppose that all summands $c_{i} x^{i}$ have value $\geq \frac{1}{p-1} v p$. Then part d) of Corollary 2.11] shows that we may replace every monomial of the form $c_{i} x^{i}$ with $i \in p \mathbb{Z}$ by the monomial $-p \tilde{c}_{i} x^{i / p}$, where $\tilde{c}_{i} \in K$ is a $p$-th root of $c_{i}$. Note that $v c_{i} x^{i} \leq \frac{p}{p-1} v p$ implies that $v\left(-p \tilde{c}_{i} x^{i / p}\right) \leq \frac{p}{p-1} v p$. After an iterated application we may then assume in addition that $I \cap p \mathbb{Z}=\emptyset$.

It remains to prove the last assertions of the lemma. If $m \neq 0$, then $v \vartheta=\frac{m}{p} v x \notin$ $v F$. Assume that $m=0$. Then $I \neq \emptyset$ because the extension $E \mid F$ is assumed to be non-trivial. Hence there is some $j \in I$ such that $v \sum_{i \in I} c_{i} x^{i}=v c_{j} x^{j}$. We have that $j \notin p \mathbb{Z}$ : if $v c_{i} x^{i} \geq \frac{1}{p-1} v p$ for all $i \in I$, then this follows from $I \subset \mathbb{Z} \backslash p \mathbb{Z}$, and otherwise it follows from the second assertion of (4.12). By Lemma 2.9 we know that $C \in K$. Performing the transformation (2.3), we find that $\eta:=\frac{\vartheta-1}{C}$ is a root of the polynomial (2.4), with $b=\sum_{i \in I} c_{i} x^{i}$. Since $v b=v c_{j} x^{j}<\frac{p}{p-1} v p \quad$ (as $j \neq 0$, equality cannot hold), we have that $v \frac{b}{C^{p}}<0$. The polynomial $g$ in (2.4) having coefficients $d_{i}$ of value $>0$, this implies that $v \vartheta<0$. It follows that $v \vartheta^{p}<v \vartheta$ and $v \vartheta^{p}<v d_{i} \vartheta^{i}$ for $2 \leq i \leq p-1$. Consequently, $p v \vartheta=v \frac{b}{C^{p}}$ by the ultrametric triangle law. This yields

$$
v \eta=\frac{1}{p} v b C^{-p}=\frac{1}{p} v c_{j}-v C+\frac{j}{p} v x \notin v K \oplus \mathbb{Z} v x=v F .
$$

In all cases, we find as in the proof of Proposition 4.5 that $(v E: v F)=p$. 
4.2. Frobenius-closed bases. The $\operatorname{ring} R=K\left[x, x^{-1}\right]$ that we used above has the following crucial properties:

$-R$ contains $K$, and its quotient field $K(x)$ is dense in $F=K(x)^{h}$,

- $R$ admits a valuation basis $\mathcal{B}=\left\{x^{i} \mid i \in I\right\}$ over $K$, containing the element 1 , such that the values $v x^{i}, i \in I$, form a system of representatives of $v K(x)$ modulo $v K$,

- The basis $\mathcal{B}$ is Frobenius-closed, i.e., the $p$-th power of every element in $\mathcal{B}$ again lies in $\mathcal{B}$.

We will need an analogue of such rings also in the residue-transcendental case. But there, we will have to work with a henselized inertially generated function field. The main goal of this section is the proof of the following lemma:

Lemma 4.7. Let $(F \mid K, v)$ be a henselized inertially generated function field of rank 1 and transcendence degree 1 with a residue-transcendental generator. Further, assume that $\bar{K}$ is perfect of characteristic $p>0$ and relatively algebraically closed in $\bar{F}$, and that $K$ is of arbitrary characteristic and closed under $p$-th roots. Then $F$ contains a subring $R$ which satisfies:

(LFC1) $\quad R$ contains $K$ and its quotient field Quot $(R)$ is dense in $F$,

(LFC2) $\quad R$ admits a valuation basis $\mathcal{B}=\left\{u_{j} \mid j \in J\right\}$ over $K$ of elements of value 0 and containing the element 1 , whose residues $\overline{u_{j}}, j \in J$, form a basis of $\bar{F} \mid \bar{K}$,

(LFC3) $\quad \mathcal{B}$ is Frobenius-closed.

The valuation basis $\mathcal{B}$ of this lemma may be called a lifting of a Frobeniusclosed basis (LFC) in view of part a) of the following lemma. Part b) is the crucial property of Frobenius-closed bases of function fields that we are going to exploit.

Lemma 4.8. Assume that Properties (LFC2) and (LFC3) hold. Then:

a) The basis $\overline{\mathcal{B}}$ of $\bar{F} \mid \bar{K}$ consisting of all $\bar{u}_{j}, j \in J$, is also Frobenius-closed. If $\bar{u}_{m}=\bar{u}_{n}^{p}$, then $u_{m}=u_{n}^{p}$.

b) If the sum

$$
\sum_{i \in I} \bar{c}_{i} \bar{u}_{i}, \quad \bar{c}_{i} \in \bar{K}, I \subset J \text { finite }
$$

is a $p$-th power, then for every $i \in I$ with $\bar{c}_{i} \neq 0$, the basis element $\bar{u}_{i}$ is a $p$-th power of a basis element.

c) If

$$
0 \neq \vartheta^{p}-\vartheta=\sum_{i \in I} \bar{c}_{i} \bar{u}_{i}, \quad \bar{c}_{i} \in \bar{K}, I \subset J \text { finite }
$$

then there is some $i \in I$ with $\bar{c}_{i} \neq 0$ such that the basis element $\bar{u}_{i}$ is a $p$-th power of a basis element.

Proof. a): Since $\mathcal{B}$ is Frobenius-closed, every $u_{j}^{p}$ is an element of $\mathcal{B}$. Hence $\bar{u}_{j}^{p}=$ $\overline{u_{j}^{p}} \in \overline{\mathcal{B}}$, which shows that $\overline{\mathcal{B}}$ is Frobenius-closed. If $\bar{u}_{m}=\bar{u}_{n}^{p}$, then $v\left(u_{m}-u_{n}^{p}\right)>$ $0=v u_{m}$, which is only possible if $u_{m}=u_{n}^{p}$ since $\mathcal{B}$ is assumed to be a valuation basis.

b): Assume that the sum is equal to

$$
\left(\sum_{j \in J_{0}} \bar{d}_{j} \bar{u}_{j}\right)^{p}, \bar{d}_{j} \in \bar{K}
$$


where $J_{0} \subset J$ is a finite index set. Then

$$
\sum_{i \in I} \bar{c}_{i} \bar{u}_{i}=\sum_{j \in J_{0}} \bar{d}_{j}^{p} \bar{u}_{j}^{p} .
$$

By part a), the elements $\bar{u}_{j}^{p}$ are also basis elements. Hence every $\bar{u}_{i}$ which appears on the left hand side (i.e., $\bar{c}_{i} \neq 0$ ) equals a $p$-th power $\bar{u}_{j}^{p}$ appearing on the right hand side.

c): Similar to b), hence left to the reader.

We also deduce the following analogue of Lemma 4.4

Lemma 4.9. Let $F$ be henselian of rank 1. Then the properties (LFC1) and (LFC2) imply that $R$ is dense in $F$. If char $K=p$, then $F=R+\wp(F)$.

Proof. In view of (LFC1), we have to show that $R$ is dense in its quotient field, for which it suffices to show that for every $r \in R$ and each $\alpha \in v F$ there is $r^{\prime} \in R$ such that $v\left(\frac{1}{r}-r^{\prime}\right)>\alpha$. Assume that there exists an element $s \in R^{\times}$with $v(r s-1)>0$ and write

$$
\frac{1}{r}=\frac{s}{1-(1-r s)}
$$

Note that $1-r s \in R$ and proceed as in the proof of Lemma 4.4. It remains to show the existence of $s$. The properties $K \subset R$ and (LFC2) imply that $v R=v F$ and $\bar{R}=\bar{F}$. Hence there is some $s_{1} \in R$ such that $\operatorname{vr} s_{1}=0$ and that the residue of the element $r s_{1} \in R$ has an inverse in $\bar{R}$, say $\overline{s_{2}}$ for suitable $s_{2} \in R$. Then the element $s=s_{1} s_{2}$ has the desired property since $\operatorname{vr} s_{1} s_{2}=v r s_{1}=0$ and $\overline{r s_{1} s_{2}}=\overline{1}$.

The last assertion follows as in the proof of Lemma 4.4.

We will now prove Lemma 4.7. Since $\bar{K}$ is assumed to be perfect and $\bar{F} \mid \bar{K}$ a function field of transcendence degree 1 with $\bar{K}$ relatively algebraically closed in $\bar{F}$, Theorem 10 of [K5] shows that there exists a Frobenius-closed basis of $\bar{F} \mid \bar{K}$. We have to lift this basis to a Frobenius-closed basis of $F$ over $K$. Recall that $F$ is of the form (4.3). Let $f(x, y)=0$ be the irreducible equation for $x, y$ over $K$, normed such that $f$ has coefficients of value $\geq 0$ with $\bar{f}(X, Y) \neq 0$. By (4.3), the polynomials $f(X, Y)$ and $\bar{f}(X, Y)$ have the same degree in $Y$, and

$$
\frac{\partial \bar{f}}{\partial Y}(\bar{x}, \bar{y}) \neq 0
$$

- The case of char $K=p$. Since $K$ is assumed to be henselian and perfect, it contains a field of representatives for the residue field $\bar{K}$ (we leave the easy proof to the reader). We identify this field with $\bar{K}$, so that we can write

$$
\bar{K} \subset K, \quad \text { with } \bar{a}=a \text { for all } a \in \bar{K} .
$$

This embedding can be extended to an embedding of $\bar{F}$ in $F$ as follows. By (4.14), we can view the polynomial $\bar{f}(x, Y)$ as a polynomial over $K(x) \subset F$. From (4.13) it follows by Hensel's Lemma that this polynomial in $Y$ has exactly one zero $y^{\prime} \in F$ with $\overline{y^{\prime}}=\bar{y}$. We have $K(x)^{h}\left(y^{\prime}\right) \subset K(x)^{h}(y)$. Again from (4.13) it follows that the polynomial $f(x, Y)$ has exactly one root in $K(x)^{h}\left(y^{\prime}\right)$ whose residue is equal to $\overline{y^{\prime}}=\bar{y}$. This root must be $y$, hence $y \in K(x)^{h}\left(y^{\prime}\right)$, and we have shown that

$$
K(x)^{h}\left(y^{\prime}\right)=K(x)^{h}(y)=F .
$$


The residue map induces on $\bar{K}$ the identity and an isomorphism

$$
\bar{K}(x) \longrightarrow \bar{K}(\bar{x}),
$$

since both $x$ and $\bar{x}$ are transcendental over $\bar{K}$. It leaves the coefficients of the irreducible polynomial $\bar{f}(X, Y)$ fixed and sends the zero $\left(x, y^{\prime}\right)$ of $\bar{f}(X, Y)$ to the zero $(\bar{x}, \bar{y})$; hence it induces an isomorphism

$$
\bar{K}\left(x, y^{\prime}\right) \longrightarrow \bar{K}(\bar{x}, \bar{y})=\bar{F} .
$$

By this isomorphism we identify

$$
x=\bar{x}, \quad y^{\prime}=\bar{y}
$$

such that

$$
\bar{F} \subset F, \quad F=(K . \bar{F})^{h},
$$

the latter being a consequence of (4.15).

By construction, $K$ and $\bar{F}$ are linearly disjoint over $\bar{K}$. We form the subring generated by both fields in $F$ :

$$
R=K[\bar{F}] \subset F .
$$

By (4.16), $F$ is the henselization of the quotient field of $R$. Since the rank of $F$ is 1 , the field $\operatorname{Quot}(R)$ is dense in its henselization by Lemma 2.4 hence $R$ satisfies property (LFC1).

Every $\bar{K}$-basis of $\bar{F}$ is at the same time a $K$-basis of $R$. As the residue map induces the identity on $\bar{F}$, every such basis is a valuation basis of $R$ over $K$. Thus, $R$ satisfies (LFC2). If we choose, as indicated above, a Frobenius-closed basis of $\bar{F} \mid \bar{K}$, then $R$ together with this basis also satisfies property (LFC3).

We summarize what we have proved:

Lemma 4.10. In the case of char $K=p$ there exists an embedding of the residue field $\bar{F}$ in $F$ respecting the residue map such that $\bar{K}=K \cap \bar{F}$, that $K$ is linearly disjoint from $\bar{F}$ over $\bar{K}$ and that $F=(K \cdot \bar{F})^{h}$. The ring $R=K[\bar{F}] \subset F$ satisfies properties (LFC1), (LFC2) and (LFC3).

- The case of char $K=0$. In this case, $K$ contains $\mathbb{Q}$ and its valuation induces the $p$-adic valuation $v_{p}$ on $\mathbb{Q}$. Since $K$ is algebraically closed, it contains a subfield $K_{0}$ such that $\overline{K_{0}}=\bar{K}$ and $v K_{0}=v_{p} \mathbb{Q}=\mathbb{Z} v p$; this field can be constructed as follows. Take $\mathcal{T}$ to be a set of preimages for a transcendence basis $\overline{\mathcal{T}}$ of $\bar{K} \mid \mathbb{F}_{p}$. Then $\mathcal{T}$ is a standard algebraically valuation independent set, and we have $\overline{\mathbb{Q}(\mathcal{T})}=\mathbb{F}_{p}(\overline{\mathcal{T}})$ and $v \mathbb{Q}(\mathcal{T})=v \mathbb{Q}$ by Lemma 2.5. Now $\bar{K} \mid \overline{\mathbb{Q}(\mathcal{T})}$ is an algebraic extension which can be viewed as a (transfinite) tower of finite separable and finite purely inseparable extensions. By induction we succesively lift all of these extensions, preserving their degrees. The separable extensions are lifted by Hensel's Lemma, using our assumption that $(K, v)$ is henselian. The purely inseparable extensions can be lifted using our assumption that $K$ is closed under $p$-th roots. We obtain a tower of finite extensions, starting from the field $\mathbb{Q}(\mathcal{T})$. Since all of them have the same degree as the corresponding extensions of their residue fields, the fundamental inequality shows that they preserve the value group as $\mathbb{Q}(\mathcal{T})$, which is $\mathbb{Z} v p$. The union over this tower is the desired field $K_{0}$. 
Note that $\overline{K_{0}(x)}=\bar{K}(\bar{x})$. So we may choose a polynomial $g(X, Y) \in K_{0}[X, Y]$ with coefficients of value $\geq 0$ such that $g$ has the same degree in $Y$ as $f$, and

$$
\bar{g}(X, Y)=\bar{f}(X, Y) \text { and } \overline{g(x, Y)}=\bar{f}(\bar{x}, Y) .
$$

From (4.13) it follows by Hensel's Lemma that $g(x, Y)$ has exactly one zero $y^{\prime} \in F$ with $\overline{y^{\prime}}=\bar{y}$. We have $K(x)^{h}\left(y^{\prime}\right) \subset K(x)^{h}(y)$. Again from (4.13) it follows that the polynomial $f(x, Y)$ has exactly one root in $K(x)^{h}\left(y^{\prime}\right)$ whose residue is equal to $\overline{y^{\prime}}=\bar{y}$. This root must be $y$, hence $y \in K(x)^{h}\left(y^{\prime}\right)$, and we have shown that

$$
K(x)^{h}\left(y^{\prime}\right)=K(x)^{h}(y)=F .
$$

Hence we assume from now on that $y$ is algebraic over $K_{0}(x)$ with

$$
\left[K_{0}(x, y): K_{0}(x)\right]=\left[\overline{K_{0}}(\bar{x}, \bar{y}): \overline{K_{0}}(\bar{x})\right]=[\bar{K}(\bar{x}, \bar{y}): \bar{K}(\bar{x})]=[K(x, y): K(x)] .
$$

In particular, this shows that the function field $F_{0}:=K_{0}(x, y)$ is linearly disjoint from $K$ over $K_{0}$. Moreover, we have

$$
\overline{F_{0}}=\overline{K_{0}}(\bar{x}, \bar{y})=\bar{F}
$$

and, in view of Lemma 2.3

$$
F=K(x)^{h}(y)=K(x, y)^{h}=\left(K . K_{0}(x, y)\right)^{h}=\left(K . F_{0}\right)^{h} .
$$

Now we lift the Frobenius-closed basis $\overline{\mathcal{B}}$ of $\bar{F} \mid \bar{K}$ to $F_{0}$. First we observe that every basis element $\bar{u} \neq 1$ in $\overline{\mathcal{B}}$ is an element of $\bar{F} \backslash \bar{K}$ and hence transcendental over $\bar{K}$, as $\bar{K}$ is assumed to be relatively algebraically closed in $\bar{F}$. This implies that there exists an integer $\nu=\nu(\bar{u})$ such that $\bar{u} \notin \bar{F}^{p^{\nu}}$. Consequently,

$$
\overline{\mathcal{B}}=\{1\} \cup\left\{\bar{u}^{p^{n}} \mid n \in \mathbb{N} \text { and } \bar{u} \in \overline{\mathcal{B}} \backslash \bar{F}^{p}\right\} .
$$

For every $\bar{u} \in \overline{\mathcal{B}} \backslash \bar{F}^{p}$ we choose an element $u \in F_{0}$ with residue $\bar{u}$. Let $\mathcal{B}^{\prime}$ be the collection of all these elements $u$. Then

$$
\mathcal{B}=\{1\} \cup\left\{u^{p^{n}} \mid n \in \mathbb{N} \text { and } u \in \mathcal{B}^{\prime}\right\}
$$

is a valuation independent set and a set of representatives for $\mathcal{B}$. Let

$$
R_{0}=K_{0}[\mathcal{B}]
$$

be the subring of $F_{0}$ generated over $K_{0}$ by the elements from $\mathcal{B}$. Since

$$
v R_{0}=v_{p} \mathbb{Q}=v F_{0} \text { and } \overline{R_{0}}=\bar{F}=\overline{F_{0}}
$$

and since the value group $v_{p} \mathbb{Q}$ is isomorphic to $\mathbb{Z}$, we conclude that $R_{0}$ is dense in $F_{0}$.

We form the subring generated by $K$ and $R_{0}$ in $F$ :

$$
R:=K\left[R_{0}\right]=K[\mathcal{B}] \subset F .
$$

Since $R_{0}$ is dense in $F_{0}$ and $K$ is of rank 1 by assumption, $R$ is dense in the ring

$$
R^{\prime}:=K\left[F_{0}\right] \subset F .
$$

From (4.17) it follows that $F$ is the henselization of the quotient field of $R^{\prime}$. Since the rank of $F$ is 1 , the field Quot $\left(R^{\prime}\right)$ is dense in its henselization by Lemma 2.4 and the fact that $R$ is dense in $R^{\prime}$ implies that $\operatorname{Quot}(R)$ is dense in $\operatorname{Quot}\left(R^{\prime}\right)$. Hence $R$ satisfies (LFC1). 
By construction, $\mathcal{B}$ is a valuation basis of $R$ over $K$, containing 1 , closed under $p$-th powers and such that $\overline{\mathcal{B}}$ is a Frobenius-closed basis of $\bar{F} \mid \bar{K}$. Hence $R$ satisfies (LFC2) and (LFC3). We summarize what we have proved:

Lemma 4.11. In the case of char $K=0$ there exists a subfield $K_{0}$ of $K$ and a function field $F_{0} \mid K_{0}$ linearly disjoint from $K \mid K_{0}$ such that $v F_{0}=v K_{0}$ is discrete, $\overline{K_{0}}=\bar{K}$ and $\overline{F_{0}}=\bar{F}$. The field $F_{0}$ contains a valuation independent set $\mathcal{B}$ including 1 and closed under $p$-th powers such that $\overline{\mathcal{B}}$ is a Frobenius-closed basis of $\bar{F}$ over $\bar{K}$. The ring $K_{0}[\mathcal{B}]$ is dense in $F_{0}$, and the ring $R=K[\mathcal{B}]$ satisfies properties (LFC1), (LFC2) and (LFC3).

\subsection{The residue-transcendental case.}

- The equal characteristic case. Using Lemma 4.10, we derive the following normal form, which proves the equal characteristic residue-transcendental case of Proposition 4.1

Proposition 4.12. Let $K, F$ and $E$ be as in the residue-transcendental case of Proposition 4.1, and assume that char $K=p$. Choose a ring $R$ with Frobeniusclosed basis $\mathcal{B}$ in $F \mid K$ as in Lemma 4.10. Then either

$$
E=F(\vartheta), \text { where } \vartheta^{p}-\vartheta \in K,
$$

or

$$
E=F(\vartheta), \quad \text { where } \vartheta^{p}-\vartheta=\sum_{i \in I} c_{i} u_{i}, \quad 0 \neq c_{i} \in K, u_{i} \in \mathcal{B},
$$

with $I$ a finite index set, and such that no element $u_{i} \neq 1$ is a $p$-th power in $\mathcal{B}$, and

$$
\forall i \in I: v c_{i} u_{i}=v c_{i} \leq 0 .
$$

If $v c_{j} u_{j}<0$ for some $j \in I$ with $u_{j} \neq 1$, then $\bar{E} \mid \bar{F}$ is purely inseparable of degree p. If $v c_{i} u_{i}=0$ for all $i \in I$, then $\bar{E} \mid \bar{F}$ is separable of degree $p$. In both cases, $(E \mid F, v)$ is defectless.

Suppose that $u_{\ell}=1$ for some $\ell \in I$ and $c_{\ell} u_{\ell}$ is the only summand of value $<0$. Take $L$ to be the Galois extension of $K$ generated by a root of the polynomial $X^{p}-X-c_{\ell}$. If $I=\{\ell\}$, then (4.18) holds and the extension $(L . E \mid L . F, v)$ is trivial. Otherwise, $\overline{L . E} \mid \overline{L . F}$ is separable of degree p. In both cases, $($ L.E $\mid L . F, v)$ is defectless, and if $(E \mid F, v)$ has non-trivial defect, then $(L \mid K, v)$ has non-trivial defect.

Proof. We can assume that $E \mid F$ is of the form (4.4). By Lemma 4.9 and (4.5), we can also assume that

$$
a=\sum_{i \in I} c_{i} u_{i}, \quad \text { where } c_{i} \in K, u_{i} \in \mathcal{B}
$$

with $I$ a finite index set and such that $v c_{i} u_{i} \leq 0$ for all $i \in I$. Note that by (LFC2),

$$
v a=\min _{i \in I} v c_{i} u_{i} \leq 0 .
$$

After a replacement procedure similar to the one used in the proof of Proposition 4.5, we can assume that no element $u_{i} \neq 1$ is a $p$-th power of another element in $\mathcal{B}$ (which by part b) of Lemma 4.8 implies that $\overline{u_{i}}$ is not a $p$-th power in $\overline{\mathcal{B}}$ ). Note that if $u_{i} \neq 1$, then $u_{i} \in F \backslash K$, which shows that there exists an integer $\nu=\nu\left(u_{i}\right)$ such that $u_{i} \notin F^{p^{\nu}}$, and so $u_{i} \notin \mathcal{B}^{p^{\mu}}$. 
Assume that $v c_{j} u_{j}<0$ for some $j \in I$ with $u_{j} \neq 1$, and choose $j$ such that

$$
v c_{j} u_{j}=\min _{i \in I, u_{i} \neq 1} v c_{i} u_{i}<0 .
$$

If $u_{\ell}=1$ for some $\ell \in I$, then as we did for the element $c_{0}$ in the proof of Proposition 4.5. we can replace $c_{\ell} u_{\ell}=c_{\ell} \in K$ by a suitable $p^{\nu}$-th root in $K$ whose value is larger than $v c_{j} u_{j}$. We can thus assume that $v c_{j} u_{j}=\min _{i \in I} v c_{i} u_{i}$. As in the proof of Proposition 4.5 we find that $v \vartheta=\frac{1}{p} v c_{j} u_{j}=\frac{1}{p} v c_{j}$. Set $d=c_{j}^{-1 / p} \in K$ and $\chi:=d \vartheta$; then $v \chi=0$ and $v d>0$. We obtain

$$
\chi^{p}-d^{p-1} \chi=d^{p}\left(\vartheta^{p}-\vartheta\right)=c_{j}^{-1} \sum_{i \in I} c_{i} u_{i} .
$$

Set $d_{i}=c_{i} / c_{j}$; then $v d_{i} \geq 0$ and $d_{j}=1$. Since $v d^{p-1} \chi>0$, we see that

$$
\bar{\chi}^{p}=\overline{\sum_{i \in I} d_{i} u_{i}}=\sum_{i \in I} \bar{d}_{i} \bar{u}_{i} .
$$

Since $\bar{d}_{j}=1$ and $\bar{u}_{j} \neq 1$ is not a $p$-th power in $\overline{\mathcal{B}}$ by construction, we can infer from part b) of Lemma 4.8 that $\bar{\chi} \notin \bar{F}$. Thus, $[\bar{E}: \bar{F}] \geq p$. By the fundamental inequality (1.1), equality holds. Since the extension is generated by $\bar{\vartheta}$, it is purely inseparable.

Assume that all summands $c_{i} u_{i}$ have value 0 . Then also $\vartheta$ has value 0 , and

$$
\bar{\vartheta}^{p}-\bar{\vartheta}=\sum_{i \in I} \bar{c}_{i} \bar{u}_{i}
$$

If the polynomial $X^{p}-X-\sum \bar{c}_{i} \bar{u}_{i}$ were reducible, then Hensel's Lemma would yield that $[E: F]<p$ in contradiction to our assumption. Hence $[\bar{E}: \bar{F}] \geq p$, and as before it follows that equality holds. As the extension is generated by $\bar{\vartheta}$, it is separable.

Assume that $u_{\ell}=1$ for some $\ell \in I$ and $c_{\ell} u_{\ell}$ is the only summand of value $<0$. Choose $\vartheta_{0} \in \tilde{K}$ such that $\vartheta_{0}^{p}-\vartheta_{0}=c_{\ell}$, and set $L=K\left(\vartheta_{0}\right)$. Then for $\vartheta_{1}:=\vartheta-\vartheta_{0} \in L . E$, we obtain from (4.5) that

$$
L . E=L . F\left(\vartheta_{1}\right), \quad \text { where } \vartheta_{1}^{p}-\vartheta_{1}=\sum_{i \in I \backslash\{\ell\}} c_{i} u_{i} .
$$

If the polynomial $X^{p}-X-\sum_{\ell \neq i \in I} \bar{c}_{i} \bar{u}_{i}$ is reducible, then it splits completely. But by part c) of Lemma 4.8, this is only possible if the sum is empty, that is, $I=\{\ell\}$. In this case, (4.18) holds with $\vartheta^{p}-\vartheta=c_{\ell}$. If the polynomial is irreducible, then $[\overline{L . E}: \overline{L . F}] \geq p$, and again, equality holds. As the extension $\overline{L . E} \mid \overline{L . F}$ is generated by $\bar{\vartheta}_{1}$, it is separable.

Finally, it remains to prove the very last assertion of the lemma. Since $\bar{K}$ is assumed to be perfect and relatively algebraically closed in $\bar{F}$, the extension $\bar{F} \mid \bar{K}$ is regular. Since also $v F=v K$, we know from Lemma 2.20 that $(F \mid K, v)$ is valuation regular. As in the proof of Proposition 4.5 it follows that if $(L . F \mid F, v)$ has non-trivial defect, then $(L \mid K, v)$ has non-trivial defect. Suppose that $(E \mid F, v)$ has non-trivial defect. Since $E \mid F$ is a subextension of $L . E \mid F$, it then follows from Lemma 2.13 that $(L . E \mid F, v)$ has non-trivial defect. Since $(L . E \mid L . F, v)$ is defectless, it follows again from Lemma 2.13 that $(L . F \mid F, v)$, and hence also $(L \mid K, v)$ has non-trivial defect. 
- The mixed characteristic case. Using Lemma 4.11, we derive the following normal form, which proves the mixed characteristic residue-transcendental case of Proposition 4.1:

Proposition 4.13. Let $K, F$ and $E$ be as in the residue-transcendental case of Proposition 4.1, and assume that char $K=0$. Choose a ring $R$ with Frobeniusclosed basis $\mathcal{B}$ in $F \mid K$ as in Lemma 4.11, Then

$$
E=F(\vartheta) \text {, where } \vartheta^{p}=r u,
$$

such that $r \in R$ has value 0 and either $r=1$ or $\bar{r} \notin \bar{F}^{p}$, and such that $u \in R$ is a 1-unit of the form

$$
u=1+\sum_{i \in I} c_{i} u_{i}, \quad c_{i} \in K, 1 \neq u_{i} \in \mathcal{B},
$$

where $I$ is a finite index set and

$$
\forall i \in I: 0<v c_{i} u_{i}=v c_{i} \leq \frac{p}{p-1} v p \quad \text { and }\left(u_{i} \in \mathcal{B}^{p} \Rightarrow v c_{i}>v p\right) .
$$

If $v c_{i} \geq \frac{1}{p-1} v p$ for all $i \in I$, then it may be assumed that no $u_{i}$ at all appearing in the sum is a $p$-th power in $\mathcal{B}$.

In all cases, $[\bar{E}: \bar{F}]=p$, with the extension being separable if and only if $r=1$ and $v c_{i}=\frac{p}{p-1} v p$ for all $i \in I$.

Proof. We can assume that $E \mid F$ is of the form (4.7). Since $v F=v K$ we can write $a=c b$, where $c \in K$ with $v c=v a$. Using (4.8) and our assumption that $K$ is closed under $p$-th roots, we may replace $a$ by $b$. Now $v b=0$, so $\bar{b} \neq 0$. If $\bar{b}$ is a $p$-th power in $\bar{F}$, then we may choose $b_{0} \in F$ with $\bar{b}_{0}=\bar{b}^{1 / p}$ and write $b=b_{0}^{p} u$ with $u$ a 1-unit. As before, we may then replace $b$ by $u$. Now suppose that $\bar{b}$ is not a $p$-th power in $\bar{F}$. By (LCF2) we can choose $r \in R$ such that $\bar{r}=\bar{b}$ and write $b=r u$ with $u$ a 1-unit. In summary, we have shown that we can assume $\vartheta^{p}=r u$ with $r \in R$ of value 0 and either $r=1$ or $\bar{r} \notin \bar{F}^{p}$, and $u \in F$ a 1-unit.

In view of Lemma 4.9 and part a) of Corollary 2.11, we may further assume that

$$
u=1+\sum_{i \in I} c_{i} u_{i} \quad \text { with } \quad c_{i} \in K, u_{i} \in \mathcal{B}, v c_{i} u_{i} \leq \frac{p}{p-1} v p \text { and } I \subset \mathbb{Z} \text { finite. }
$$

Since $u$ is a 1 -unit, we have $v \sum c_{i} u_{i}>0$, and since the elements $u_{i}$ form a valuation basis of $R$ over $K$ by (LFC2), we find that $v c_{i} u_{i}>0$ for each $i \in I$.

All further properties stated for the summands $c_{i} u_{i}$ in the proposition are now obtained by a replacement procedure as in the proof of Proposition 4.6 $\bar{F}$.

It remains to prove the last assertions of the lemma. If $\bar{r} \notin \bar{F}^{p}$, then $\bar{\vartheta}=\bar{r}^{1 / p} \notin$

Now assume that $r=1$. By Lemma 2.9 we know that $C \in K$. Performing the transformation (2.3), we find that

$$
\eta:=\frac{\vartheta-1}{C}
$$

is a root of the polynomial (2.4), where $b=\sum_{i \in I} c_{i} u_{i}$. Suppose first that $v c_{i} u_{i}=$ $\frac{p}{p-1} v p$ for all $i \in I$. Then $v b C^{-p}=0$, and the residue polynomial $Z^{p}-Z-\overline{b C}-p$ does not admit a zero in $\bar{F}$ since otherwise $\eta \in F$ by Hensel's Lemma and $E \mid F$ would be trivial, contrary to our assumption that its degree is $p$. 
Now suppose that $v c_{i} u_{i}<\frac{p}{p-1} v p$ for some $i \in I$. Then for a suitable $j \in I$,

$$
v b=\min _{i \in I} v c_{i} u_{i}=v c_{j}<\frac{p}{p-1} v p
$$

and $v b C^{-p}<0$. As in the proof of Proposition 4.6 we find that

$$
v \eta=\frac{1}{p} v b C^{-p}=\frac{1}{p} v c_{j}-v C<0 .
$$

By our assumption on $K$ there is some $d \in K$ such that $d^{p}=c_{j}^{-1}$. We have that $v d C \eta=0$ and $v d C>0$. Hence for $\chi:=d C \eta$ we obtain

$$
\chi^{p}-(d C)^{p-1} \chi=(d C)^{p}\left(\eta^{p}-\eta\right)=(d C)^{p} \frac{b}{C^{p}}=c_{j}^{-1} \sum_{i \in I} c_{i} u_{i} .
$$

As in Proposition 4.12 we see that $\bar{\chi} \notin \bar{F}$.

In all cases, we find that $[\bar{E}: \bar{F}] \geq p$ and, hence, $[\bar{E}: \bar{F}]=p$ by the fundamental inequality. It follows that $\bar{E} \mid \bar{F}$ is a separable extension (generated by a root of an Artin-Schreier polynomial) if $r=1$ and $v c_{i}=\frac{p}{p-1} v p$ for all $i \in I$, and that it is a purely inseparable extension in the remaining cases.

\section{Proof of the generalized stability theorem}

We prove Theorem 1.1 by a stepwise reduction to the analysis of Galois extensions of degree $p$ of certain valued function fields without transcendence defect, followed by an application of the results of Section 4 Since every valued field $(K, v)$ of residue characteristic 0 is a defectless field, we will assume that char $K v=p>0$.

\section{- Reduction to transcendence degree 1.}

Lemma 5.1. To prove Theorem 1.1, it suffices to prove

(R1) Every valued function field of transcendence degree 1 without transcendence defect over a defectless ground field is a defectless field.

Proof. By induction on the transcendence degree of the function field. The case of transcendence degree 1 is covered by $(\mathbf{R} 1)$. Assume that $(F \mid K, v)$ is a valued function field of transcendence degree $>1$ without transcendence defect. Choose any subfunction field $F_{0} \mid K$ in $F \mid K$ such that $0<\operatorname{trdeg} F_{0}|K<\operatorname{trdeg} F| K$. By the additivity of transcendence degree and rational rank, both $\left(F_{0} \mid K, v\right)$ and $\left(F \mid F_{0}, v\right)$ are valued function fields without transcendence defect. Hence if $(K, v)$ is a defectless field, then by induction hypothesis $\left(F_{0}, v\right)$, and consequently $(F, v)$ is also a defectless field.

\section{- Reduction to algebraically closed ground fields.}

Lemma 5.2. To prove (R1), it suffices to prove

(R2) Every valued function field of transcendence degree 1 without transcendence defect over an algebraically closed field is a defectless field.

Proof. Let $(F \mid K, v)$ satisfy the assumptions of (R1). We pick a valuation-transcendental element $t \in F$. By Lemma 2.22, $(K(t) \mid K, v)$ is a valuation regular extension, and so is $(\tilde{K}(t) \mid \tilde{K}, v)$ under every extension of $v$ to $\tilde{K}(t)$. Hence by (R2), $(\tilde{K}(t), v)$ is a defectless field. From Corollary 2.25 we infer that the same holds for $(K(t), v)$. Since $F \mid K(t)$ is finite, it follows from Corollary 2.16 that $(F, v)$ is also a defectless field. 


\section{- Reduction to finite rank.}

Lemma 5.3. To prove (R2), it suffices to prove

(R3) Every valued function field of transcendence degree 1 without transcendence defect over an algebraically closed ground field of finite rank is a defectless field.

Proof. Let $(F \mid K, v)$ satisfy the assumptions of (R2). We wish to show that $(F, v)$ is a defectless field. As in the preceding proof, we choose a valuation transcendental element $t$, and by Corollary 2.16 we only have to show that $(K(t), v)$ is a defectless field. By Theorem 2.14 we may as well show that $\left(K(t)^{h}, v\right)$ is a defectless field.

Let $\left(K(t)^{h}\left(a_{1}, \ldots, a_{n}\right) \mid K(t)^{h}, v\right)$ be an arbitrary finite extension; we have to show that it is defectless. There exists a finitely generated extension $k_{1}$ of the prime field of $K$ such that $a_{1}, \ldots, a_{n}$ are already algebraic over $k_{1}(t)$. We take $k$ to be the algebraic closure of $k_{1}$ inside $K$. Since $k_{1}$ is finitely generated over its prime field, the rank of $\left(k_{1}, v\right)$ and thus also of its algebraic closure $(k, v)$ must be finite by Corollary 2.7. By construction, $(k(t) \mid k, v)$ is a valued function field of transcendence degree 1 without transcendence defect over the algebraically closed ground field $(k, v)$ of finite rank. So (R3) together with Theorem 2.14 implies that $\left(k(t)^{h}\left(a_{1}, \ldots, a_{n}\right) \mid k(t)^{h}, v\right)$ is a defectless extension.

Since $k$ is algebraically closed, Lemma 2.20 shows that the extension $(K \mid k, v)$ is valuation regular. By Lemma 2.23 and Corollary 2.21, the extensions $(K(t) \mid k(t), v)$ and $\left(K(t)^{h} \mid k(t)^{h}, v\right)$ are also valuation regular. Hence the algebraic extension $\left(k(t)^{h}\left(a_{1}, \ldots, a_{n}\right) \mid k(t)^{h}, v\right)$ is valuation disjoint from $\left(K(t)^{h} \mid k(t)^{h}, v\right)$. Now we apply Proposition 2.24 to obtain that

$$
\mathrm{d}\left(K(t)^{h}\left(a_{1}, \ldots, a_{n}\right) \mid K(t)^{h}, v\right) \leq \mathrm{d}\left(k(t)^{h}\left(a_{1}, \ldots, a_{n}\right) \mid k(t)^{h}, v\right)=1 .
$$

We have thus proved that $\left(K(t)^{h}, v\right)$ is a defectless field.

\section{- Reduction to rank 1.}

Lemma 5.4. To prove (R3), it suffices to prove

(R4) Every valued function field of rank 1 and of transcendence degree 1 without transcendence defect over an algebraically closed ground field is a defectless field.

Proof. Let $(F, v)$ satisfy the assumptions of (R3). Then $(F, v)$ must also have finite rank (cf. (1.2) and note that the rank of $v F$ cannot exceed the rank of $v K$ plus $\operatorname{rr} v L / v K)$. Let $v=w_{1} \circ \ldots \circ w_{n}$ be the decomposition of $v$ into valuations $w_{i}$ of rank 1. By Lemma 2.1, every $K w_{1} \circ \ldots \circ w_{i}$ is an algebraically closed field. By a repeated application of Lemma 2.8 $\left(F \mid K, w_{1}\right)$ and all $\left(F w_{1} \circ \ldots \circ w_{i} \mid K w_{1} \circ \ldots \circ w_{i}, w_{i+1}\right)$ are valued function fields without transcendence defect. Hence (R4) yields that every $\left(F w_{1} \circ \ldots \circ w_{i}, w_{i+1}\right)$ is a defectless field. Now a repeated application of Lemma 2.17 shows that $(F, v)$ itself is a defectless field.

To complete our proof, we show that $(\mathrm{R} 4)$ is true. Let $(F \mid K, v)$ satisfy the conditions of (R4). Then there is a valuation-transcendental element $x \in F$ and $F \mid K(x)$ is finite. By Corollary 2.16 it suffices to prove (R4) under the additional assumption that $F \mid K$ is rational with a valuation-transcendental generator. In view of Theorem 2.14 we can replace $(F, v)$ by its henselization. More generally, we will now prove our assertion under the assumption that $(F \mid K, v)$ is a henselized inertially generated function field of rank 1 and transcendence degree 1 with a 
valuation-transcendental generator over the algebraically closed field $K$. Given an arbitrary finite extension $(E \mid F, v)$, we have to show that it is defectless.

Since the ramification group is a $p$-group (cf. [En]), $F^{\mathrm{sep}} \mid F^{r}$ is a $p$-extension. It follows from the general theory of $p$-groups (cf. $[\underline{\mathrm{H}}$, Chapter III, $\S 7$, Satz 7.2 and the following remark) via Galois correspondence that the maximal separable subextension of $E . F^{r} \mid F^{r}$ is a finite tower of Galois extensions of degree $p$. Consequently, $E . F^{r} \mid F^{r}$ is a finite tower of normal extensions of degree $p$, either Galois or purely inseparable. Then there is already a finite subextension $N \mid F$ of $F^{r} \mid F$ such that $E . N \mid N$ is such a tower. Lemma 2.27 shows that $N$, being a finite subextension inside $F^{r}$, is again a henselized inertially generated function field of transcendence degree 1 with a valuation-transcendental generator over $K$. Also, it is again of rank 1. By Proposition 2.18 we have $\mathrm{d}(E \mid F, v)=\mathrm{d}(E \cdot N \mid N, v)$, hence it suffices to prove that $E . N \mid N$ is defectless. Since this is trivial if $E . N=N$, we assume that $E . N \neq N$. Then the first extension in the tower is defectless by Corollary 4.2 or Proposition 3.1. This yields $\mathrm{d}(E \mid F, v)=\mathrm{d}(E . N \mid N, v)<[E . N: N] \leq[E: F]$; that is, $(E \mid F, v)$ cannot be immediate. We have proved:

Henselized inertially generated function fields of rank 1 and of transcendence degree 1 with a valuation-transcendental generator over an algebraically closed ground field do not admit proper immediate algebraic extensions.

We use this fact to prove:

Lemma 5.5. Every henselized function field $(F, v)$ of rank 1 and of transcendence degree 1 without transcendence defect over an algebraically closed ground field $K$ is a henselized inertially generated function field with a valuation-transcendental generator.

Proof. Case I) Suppose $F$ contains a value-transcendental element $x$. Since $\overline{K(x)}=$ $\bar{K}$ is algebraically closed and $\bar{F} \mid \overline{K(x)}$ is finite, we have $\bar{F}=\overline{K(x)}$. Further, $v F$ is a finite extension of $v K(x)=v K \oplus \mathbb{Z} v x$. Since $v K$ is divisible, we have that $v F=v K \oplus \mathbb{Z} \alpha$ for some $\alpha \in v F$. Choose $x^{\prime} \in F$ such that $v x^{\prime}=\alpha$. Then $v F=v K \oplus \mathbb{Z} v x^{\prime}=v K\left(x^{\prime}\right)$ by Lemma 2.5, and $\bar{F}=\bar{K}=\overline{K\left(x^{\prime}\right)}$. Now the henselian field $F$ contains the henselization $K\left(x^{\prime}\right)^{h}$, and we have just shown that $F \mid K\left(x^{\prime}\right)^{h}$ is an immediate extension. But the henselized inertially generated function field $K\left(x^{\prime}\right)^{h}$ of rank 1 and of transcendence degree 1 with value-transcendental generator $x^{\prime}$ over $K$ does not admit any proper immediate algebraic extension. This yields $F=K\left(x^{\prime}\right)^{h}$.

Case II) Suppose $F$ contains a residue-transcendental element $x$. Since $v K(x)=$ $v K$ is divisible and $v F / v K(x)$ is finite, we have $v F=v K(x)$. Since $\bar{F} \mid \overline{K(x)}$ is finite and $\overline{K(x)}=\bar{K}(\bar{x})$ is a function field of transcendence degree 1 over $\bar{K}$, the same holds for $\bar{F}$. Since $\bar{K}$ is algebraically closed, there is a separating transcendence basis $\{\xi\}$ of $\bar{F} \mid \bar{K}$. We choose $x^{\prime} \in F$ such that $\overline{x^{\prime}}=\xi$. The henselian field $F$ contains the henselization $K\left(x^{\prime}\right)^{h}$. Since $\bar{F} \mid \bar{K}(\xi)$ is a finite separable extension, $\bar{F}=\bar{K}(\xi, \eta)$ for some $\eta$ which is separable-algebraic over $\bar{K}(\xi)$. Using Hensel's Lemma, we lift $\eta$ to an element $y^{\prime} \in F$ such that the finite subextension $K\left(x^{\prime}\right)^{h}\left(y^{\prime}\right) \mid K\left(x^{\prime}\right)^{h}$ of $F \mid K\left(x^{\prime}\right)^{h}$ has the same degree as $\bar{F} \mid \bar{K}(\xi)$ and such that $\overline{K\left(x^{\prime}\right)^{h}\left(y^{\prime}\right)}=\bar{F}$. It follows that $K\left(x^{\prime}\right)^{h}\left(y^{\prime}\right) \mid K\left(x^{\prime}\right)^{h}$ is unramified and that $\left(K\left(x^{\prime}\right)^{h}\left(y^{\prime}\right) \mid K, v\right)$ is a henselized inertially generated function field. Since $v K\left(x^{\prime}\right)^{h}\left(y^{\prime}\right)=v F$, we find that $F \mid K\left(x^{\prime}\right)^{h}\left(y^{\prime}\right)$ is immediate. But as proved above, the henselized inertially generated function field $K\left(x^{\prime}\right)^{h}\left(y^{\prime}\right)^{h}$ of rank 1 and of transcendence degree 1 with residue-transcendental 
generator over $K$ does not admit any proper immediate algebraic extension. This yields $F=K\left(x^{\prime}\right)^{h}\left(y^{\prime}\right)$.

The proof that $E . N \mid N$ is defectless now proceeds by induction on the number of extensions appearing in the tower. Since we have assumed that $E . N \neq N$, there is a normal subextension $E^{\prime} \mid N$ of $E . N \mid N$ of degree $p$. By Corollary 4.2 or Proposition 3.1. this extension is defectless. From the preceding lemma we infer that $E^{\prime}$ is again a henselized inertially generated function field of rank 1 and transcendence degree 1 with a valuation-transcendental generator over $K$. Its rank is 1 since it is an algebraic extension of $N$. By induction hypothesis, $\left(E \mid E^{\prime}, v\right)$ is also defectless since it has a smaller degree than $E \mid N$. Hence by Lemma $2.13,(E \mid N, v)$ is defectless. This completes the proof of the first assertion of Theorem 1.1.

The "inseparably defectless" version of Theorem 1.1 has already been proved in Section 3 (see Proposition 3.1). So it remains to prove the "separably defectless" version. Assume that $(K, v)$ is a separably defectless field, $(F \mid K, v)$ is a valued function field without transcendence defect, and that $v K$ is cofinal in $v F$. Then the completion $F^{c}$ of $(F, v)$ contains the completion $K^{c}$ of $(K, v)$. A valued field is separably defectless if and only if its completion is defectless (cf. [K6]). Hence, $K^{c}$ is a defectless field. We consider the subfield $F \cdot K^{c} \subset F^{c}$ which is a function field over $K^{c}$. Since $\left(K^{c} \mid K, v\right)$ and $\left(F^{c} \mid F, v\right)$ are immediate extensions and $\operatorname{trdeg} K^{c} \cdot F \mid K^{c} \leq$ $\operatorname{trdeg} F \mid K,\left(K^{c} . F \mid K^{c}, v\right)$ is again a valued function field without transcendence defect. By the "defectless" version of Theorem 1.1 it follows that $\left(K^{c} . F, v\right)$ is a defectless field. Hence also its completion $\left(F^{c}, v\right)$ is defectless. By the above cited theorem it follows that $(F, v)$ is defectless. This completes the proof of the first assertion of Theorem 1.1.

- Proof of Corollary 1.2. By Theorem 1.1, $(\tilde{K} . F, v)$ is a defectless field for every extension of the valuation $v$. Therefore, $(\tilde{K} . F, v)$ is defectless in $\tilde{K} . E$. Let $v_{1}, \ldots, v_{\mathrm{g}}$ be all extensions of $v$ from $\tilde{K} . F$ to $\tilde{K} . E$. Then there exists a finite extension $L_{0} \mid K$ such that:

1) $\left[L_{0} \cdot E: L_{0} \cdot F\right]=[\tilde{K} \cdot E: \tilde{K} \cdot F]$,

2) the restrictions of $v_{1}, \ldots, v_{\mathrm{g}}$ to $L_{0} . E$ are all distinct,

3) $\left(v_{i} L_{0} \cdot E: v_{i} L_{0} \cdot F\right) \geq\left(v_{i} \tilde{K} \cdot E: v_{i} \tilde{K} \cdot F\right)$ and $\left[L_{0} \cdot E v_{i}: L_{0} \cdot F v_{i}\right] \geq\left[\tilde{K} \cdot E v_{i}:\right.$ $\left.\tilde{K} . F v_{i}\right]$ for $1 \leq i \leq$ g. (In order to obtain the first inequality, choose representatives of the cosets of $v_{i} \tilde{K} . E / v_{i} \tilde{K} . F$ and choose $L_{0}$ so large that they lie in $v_{i} L_{0} . E$. For the second inequality, choose a basis of $\tilde{K} . E v_{i} \mid \tilde{K} . F v_{i}$ and choose $L_{0}$ so large that it is contained in $L_{0} . E v_{i}$.)

These conditions will remain true whenever $L_{0}$ is replaced by any algebraic extension $L$ of $K$ which contains $L_{0}$. Since equality holds in the fundamental inequality for the extension $\tilde{K} . E$ of $(\tilde{K} . F, v)$, the same is then true for the extension L.E of $(L . F, v)$.

Now assume in addition that $(K, v)$ is henselian. Then by a result of Pank (cf. $[\mathrm{K}-\mathrm{P}-\mathrm{R}]$ ) there exists a field complement $W$ to its absolute ramification field $K^{r}$, that is, $W \mid K$ is linearly disjoint from $K^{r} \mid K$ (and hence purely wild), and $K^{r} . W=\tilde{K}$. Since $\tilde{W}=\tilde{K}=K^{r} . W=W^{r},(W, v)$ is a defectless field. Therefore, we can replace $\tilde{K}$ by $W$ in the above and choose $L_{0} \mid K$ as a subextension of $W \mid K$ so that it is purely wild. 


\section{Proof of Corollary 1.4}

The valued function field $(\tilde{K} . F \mid \tilde{K}, v)$ satisfies the assumptions of Theorem 1.3 , so we may choose a standard valuation transcendence basis $\mathcal{T}$ of $(\tilde{K} . F \mid \tilde{K}, v)$ with $v \tilde{K} . F=v \tilde{K} \oplus \mathbb{Z} v x_{1} \oplus \ldots \oplus \mathbb{Z} v x_{r}$ and $\bar{y}_{1}, \ldots, \bar{y}_{s}$ a separating transcendence basis of $\overline{\tilde{K} . F} \mid \overline{\tilde{K}}$ such that $\left[(\tilde{K} . F)^{h}: \tilde{K}(\mathcal{T})^{h}\right]=[\overline{\tilde{K} . F}: \overline{\tilde{K}(\mathcal{T})}]$. Pick $\zeta \in \overline{\tilde{K} . F}$ such that $\overline{\tilde{K} . F}=\overline{\tilde{K}(\mathcal{T})}(\zeta)$ and take $\bar{f}$ to be its separable minimal polynomial over $\overline{\tilde{K}(\mathcal{T})}$. Then there exists a finite extension $L_{0} \mid K$ such that:

1) $\mathcal{T} \subseteq L_{0} . F$,

2) $\left[\left(L_{0} \cdot F\right)^{h}: L_{0}(\mathcal{T})^{h}\right]=\left[(\tilde{K} \cdot F)^{h}: \tilde{K}(\mathcal{T})^{h}\right]$,

3) $\overline{L_{0}}\left(\bar{y}_{1}, \ldots, \bar{y}_{s}\right)$ contains all coefficients of $\bar{f}$.

These conditions will remain true whenever $L_{0}$ is replaced by any algebraic extension $L$ of $K$ which contains $L_{0}$. For such $L$, we have that $\mathcal{T} \subset L . F$ remains a valuation transcendence basis of $(L . F \mid L(\mathcal{T}), v)$ since $L \mid K$ is algebraic. This yields that $\overline{L(\mathcal{T})}=\bar{L}\left(\bar{y}_{1}, \ldots, \bar{y}_{s}\right)$ by Lemma 2.5. Now we compute

$$
\begin{aligned}
{\left[(L . F)^{h}: L(\mathcal{T})^{h}\right] } & \geq[\overline{L . F}: \overline{L(\mathcal{T})}] \geq\left[\bar{L}\left(\bar{y}_{1}, \ldots, \bar{y}_{s}, \zeta\right): \bar{L}\left(\bar{y}_{1}, \ldots, \bar{y}_{s}\right)\right]=\operatorname{deg} \bar{f} \\
& =[\tilde{\tilde{K}} \cdot F: \overline{\tilde{K}(\mathcal{T})}]=\left[(\tilde{K} \cdot F)^{h}: \tilde{K}(\mathcal{T})^{h}\right]=\left[(L . F)^{h}: L(\mathcal{T})^{h}\right] .
\end{aligned}
$$

We see that equality must hold everywhere. In particular, the first equality shows that the extension $\left((L . F)^{h} \mid L(\mathcal{T})^{h}, v\right)$ is unramified.

\section{ACKNOWLEDGEMENTS}

The author thanks Peter Roquette for his invaluable help and support. He also thanks F. Delon, B. Green, A. Prestel, F. Pop, and R. Strebel for inspiring discussions and suggestions that helped prove the Generalized Stability Theorem. During the work on this paper, the author was partially supported by a Canadian NSERC grant. He has also been a member of the Newton Institute in Cambridge and a guest at the University of Konstanz; he gratefully acknowledges their support.

\section{REFERENCES}

[B] Bourbaki, N.: Elements of mathematics. Commutative algebra. Translated from the French. Hermann, Paris; Addison-Wesley Publ. Co., Reading, MA (1972). MR0360549 (50:12997)

[B-G-R] Bosch, S. - Güntzer, U. - Remmert, R. : Non-Archimedean Analysis, Springer-Verlag, Berlin (1984). MR746961 (86b:32031)

[En] Endler, O.: Valuation theory, Springer, Berlin (1972). MR0357379 (50:9847)

[Ep] Epp, Helmut H. P.: Eliminating Wild Ramification, Inventiones Math. 19 (1973), 235249. MR0321929 (48:294)

[G] Gruson, L. : Fibrés vectoriels sur un polydisque ultramétrique, Ann. Sci. Ec. Super., IV. Ser. 177 (1968), 45-89. MR0229654 (37:5228)

[G-M-P] Green, B. - Matignon, M. - Pop, F. : On valued function fields I, Manuscripta Math. 65 (1989), 357-376. MR1015661 (91g:12010)

[G-R] Grauert, H. - Remmert, R.: Uber die methode der diskret bewerteten ringe in der nicht archimedischen Analysis, Invent. Math. 2 (1966), 87-133. MR0206039(34:5864)

[H] Huppert, B.: Endliche Gruppen I, Springer, Berlin (1967). MR0224703 (37:302)

[K-K1] Knaf, H. - Kuhlmann, F.-V.: Abhyankar places admit local uniformization in any characteristic, Ann. Scient. Ec. Norm. Sup. 38 (2005), 833-846. MR 2216832 (2007k:13006)

[K-K2] Knaf, H. - Kuhlmann, F.-V.: Every place admits local uniformization in a finite extension of the function field, Adv. Math. 221 (2009), no. 2, 428-453. MR2508927

[K1] Kuhlmann, F.-V.: Henselian function fields and tame fields, extended version of Ph.D. thesis, Heidelberg (1990). 
[K2] Kuhlmann, F.-V.: On local uniformization in arbitrary characteristic, The Fields Institute Preprint Series, Toronto (1997).

[K3] Kuhlmann, F.-V.: Valuation theoretic and model theoretic aspects of local uniformization, in: Resolution of Singularities - A Research Textbook in Tribute to Oscar Zariski. H. Hauser, J. Lipman, F. Oort, A. Quiros (eds.), Progress in Mathematics Vol. 181, Birkhäuser-Verlag, Basel (2000), 381-456. MR.1748629 (2001c:14001)

[K4] Kuhlmann, F.-V.: A correction to Epp's paper "Elimination of wild ramification", Inventiones Math. 153 (2003), 679-681. MR2000472 (2004g:13017)

[K5] Kuhlmann, F.-V.: Additive Polynomials and Their Role in the Model Theory of Valued Fields, Logic in Tehran, 160-203, Lect. Notes Log., 26, Assoc. Symbol. Logic, La Jolla, CA, 2006. MR2262319 (2007k:03095)

[K6] Kuhlmann, F.-V.: A classification of Artin Schreier defect extensions and a characterization of defectless fields, submitted.

[K7] Kuhlmann, F.-V.: The model theory of tame valued fields, in preparation.

[K8] Kuhlmann, F.-V.: Elimination of Ramification II: Henselian Rationality, in preparation.

[K-P] Kuhlmann, F.-V. - Prestel, A.: On places of algebraic function fields, J. Reine Angew. Math. 353 (1984), 181-195. MR765832 (86d:12014)

[K-P-R] Kuhlmann, F.-V. - Pank, M. - Roquette, P.: Immediate and purely wild extensions of valued fields, Manuscripta Math. 55 (1986), 39-67. MR828410 (87d:12012)

[L] Lang, S. : Algebra, Springer, New York, 3rd. ed. (2002). MR.1878556 (2003e:00003)

[O] Ohm, J.: The Henselian defect for valued function fields, Proc. Amer. Math. Soc. 107 (1989), no. 2, 299-308. MR.975654 (90h:12012)

[R] Ribenboim, P.: Théorie des valuations, Les Presses de l'Université de Montréal, Montréal, 2nd ed. (1968). MR0249425 (40:2670)

[W] Warner, S.: Topological fields, Mathematics studies 157, North-Holland, Amsterdam (1989). MR.1002951 (90i:12012)

[Z-S] Zariski, O. - Samuel, P. : Commutative Algebra, Vol. II, The University Series in Higher Mathematics, D. Von Nostrand Co., Inc., Princeton, NJ-Toronto-London-New York. MR0120249(22:11006)

Department of Mathematics and Statistics, University of Saskatchewan, 106 Wiggins Road, Saskatoon, Saskatchewan, Canada S7N 5E6

E-mail address: fvk@math.usask.ca 